\title{
Constructing Vector-Valued Siegel Modular Forms from Scalar-Valued Siegel Modular Forms
}

\author{
Fabien Cléry and Gerard van der Geer
}

\begin{abstract}
This paper gives a simple method for constructing vector-valued Siegel modular forms from scalar-valued ones. The method is efficient in producing the siblings of Delta, the smallest weight cusp forms that appear in low degrees. It also shows the strong relations between these modular forms of different degrees. We illustrate this by a number of examples.

Keywords: Siegel modular form, vector-valued modular form.
\end{abstract}

\section{Introduction}

In this paper we describe a simple method to construct vector-valued Siegel modular forms from scalar-valued ones by developing modular forms in the normal bundle of a locus on which they vanish. If $f$ is a scalar-valued Siegel modular form of degree $g$ and weight $k$, given by a holomorphic function on the Siegel upper half space $\mathfrak{H}_{g}$ and $1 \leq j \leq g-1$ an integer, then the restriction of $f$ to the diagonally embedded $\mathfrak{H}_{j} \times \mathfrak{H}_{g-j}$ in $\mathfrak{H}_{g}$ gives a tensor product $f^{\prime} \otimes f^{\prime \prime}$ of modular forms of weight $k$ and degree $j$ and $g-j$. If $f$ vanishes on $\mathfrak{H}_{j} \times \mathfrak{H}_{g-j}$, then we can develop $f$ in the normal bundle of $\mathfrak{H}_{j} \times \mathfrak{H}_{g-j}$ inside $\mathfrak{H}_{g}$ and one finds as lowest non-zero term a sum of tensor products of vector-valued Siegel modular forms of degree $j$ and $g-j$. By applying this to well-known scalar-valued Siegel modular forms one can produce explicit vector-valued Siegel modular forms on the full group $\operatorname{Sp}(2 g, \mathbb{Z})$. In this way one can produce for example many siblings of Delta, modular forms that play for a given low degree $g$ a role analogous to the role that $\Delta$, the cusp form of weight 12 on $\mathrm{SL}(2, \mathbb{Z})$, plays for elliptic modular forms. That is, the cusp forms that appear among the first few if one orders these according to their Deligne weight. For example, for degree 2 the first cusp forms that

Received September 30, 2014.

1991 Mathematics Subject Classification. 14J15, $10 \mathrm{D}$. 
appear are a cusp form $\chi_{10}$ of weight 10 , a form $\chi_{12}$ of weight 12 and a vector-valued form $\chi_{6,8}$ of weight $(j, k)=(6,8)$ of Deligne weight $j+2 k-3$ equal to 17, 21 and 19. The forms $\chi_{10}$ and $\chi_{6,8}$ appear immediately in the development of the Schottky form, a scalar-valued cusp form of weight 8 in degree 4 , along $\mathfrak{H}_{2} \times \mathfrak{H}_{2}$. Similarly, the first cusp form in degree 3 is a cusp form of weight $(4,0,8)$ and is also obtained by developing the Schottky form, this time along $\mathfrak{H}_{1} \times \mathfrak{H}_{3}$.

Recently there has been quite some progress in our knowledge of vectorvalued Siegel modular forms of low degree; besides [3] there is the impressive work of Chenevier and Renard and Taïbi (see [5, 6, 20]) who among other things give conjectural values for the dimensions of spaces of cusp forms for degree $g \leq 6$. As an illustration of our method we construct cusp forms in a number of cases where the dimension of the space is predicted to be 1 . For example, we construct the 'first' cusp form in degree 5 , of weight $(2,0,0,0,10)$. Likewise, we construct a form of weight $(2,0,0,0,0,0,10)$ in degree 7 , again of rather low Deligne weight (44) compared with that (56) of the first scalar-valued cusp form (of weight 12).

Just like the ubiquitous $\Delta$, the siblings of $\Delta$ appear to play a role at many places, e.g. in the study of K3 surfaces, and they all appear to be intimately connected. We study the form $\chi_{6,8}$ and some other siblings of Delta in more detail, giving alternative constructions and calculating eigenvalues of Hecke operators. In an appendix we summarize some facts about Hecke operators of degree 2 and 3 that we need.

\section{Restricting scalar-valued modular forms}

Let $\Gamma_{g}=\operatorname{Sp}(2 g, \mathbb{Z})$ be the symplectic group of degree $g$ acting in the usual way on the Siegel upper half space $\mathfrak{H}_{g}$ :

$$
\tau \mapsto(a \tau+b)(c \tau+d)^{-1} \quad \text { for all } \tau \in \mathfrak{H}_{g} \text { and }\left(\begin{array}{ll}
a & b \\
c & d
\end{array}\right) \in \Gamma_{g} .
$$

We denote by $M_{k}\left(\Gamma_{g}\right)$ the space of scalar-valued Siegel modular forms of weight $k$ on $\Gamma_{g}$, that is, holomorphic functions on $\mathfrak{H}_{g}$ satisfying

$$
f\left((a \tau+b)(c \tau+d)^{-1}\right)=\operatorname{det}(c \tau+d)^{k} f(\tau)
$$

for all $(a, b ; c, d) \in \Gamma_{g}$ and $\tau \in \mathfrak{H}_{g}$ (and satisfying an additional holomorphicity condition at infinity for $g=1$ ). More generally, if $g>1$ and $\rho: \mathrm{GL}_{g} \rightarrow$ $\mathrm{GL}(V)$ denotes a finite-dimensional complex representation of $\mathrm{GL}_{g}$, then by $M_{\rho}\left(\Gamma_{g}\right)$ we mean the vector space of holomorphic functions $f: \mathfrak{H}_{g} \rightarrow V$ such 
that

$$
f\left((a \tau+b)(c \tau+d)^{-1}\right)=\rho(c \tau+d) f(\tau) .
$$

If $\rho$ is an irreducible complex representation of $\mathrm{GL}_{g}$ of highest weight $w=\left(a_{1} \geq a_{2} \geq \cdots \geq a_{g}\right)$ then we denote the complex vector space $M_{\rho}\left(\Gamma_{g}\right)$ by $M_{\mathbf{k}}\left(\Gamma_{g}\right)$ with $\mathbf{k}=\left(a_{1}-a_{2}, a_{2}-a_{3}, \ldots, a_{g-1}-a_{g}, a_{g}\right)$. The subspaces of cusp forms are denoted by $S_{k}\left(\Gamma_{g}\right)$ and $S_{\rho}\left(\Gamma_{g}\right)$. These cusp forms can be interpreted as sections of a line bundle or a vector bundle on the quotient space $\mathcal{A}_{g}=\Gamma_{g} \backslash \mathfrak{H}_{g}$.

If $f \in M_{k}\left(\Gamma_{g}\right)$ is a scalar-valued modular form of weight $k$ we can restrict $f$ to $\mathfrak{H}_{j} \times \mathfrak{H}_{g-j}$, where we use the modular embedding $\Gamma_{j} \times \Gamma_{g-j} \rightarrow \Gamma_{g}$, given by

$$
\left(\left(\begin{array}{ll}
a & b \\
c & d
\end{array}\right),\left(\begin{array}{ll}
\alpha & \beta \\
\gamma & \delta
\end{array}\right)\right) \mapsto\left(\begin{array}{llll}
a & 0 & b & 0 \\
0 & 0 & 0 & \beta \\
c & 0 & d & 0 \\
0 & \gamma & 0 & \delta
\end{array}\right)
$$

with corresponding map for the symmetric spaces

$$
\mathfrak{H}_{j} \times \mathfrak{H}_{g-j} \rightarrow \mathfrak{H}_{g}, \quad\left(\tau^{\prime}, \tau^{\prime \prime}\right) \mapsto\left(\begin{array}{cc}
\tau^{\prime} & 0 \\
0 & \tau^{\prime \prime}
\end{array}\right)
$$

with image $\mathcal{A}_{j, g-j}$ of $\mathcal{A}_{j} \times \mathcal{A}_{g-j}$ in $\mathcal{A}_{g}$. The result is a tensor product of modular forms $f^{\prime} \otimes f^{\prime \prime} \in M_{k}\left(\Gamma_{j}\right) \otimes M_{k}\left(\Gamma_{g-j}\right)$. In [17] Witt used this method to study Siegel modular form of degree 2. However, often this restriction vanishes. If this is the case we can develop $f$ in the conormal bundle of $\mathfrak{H}_{j} \times \mathfrak{H}_{g-j}$ in $\mathfrak{H}_{g}$. This conormal bundle is a vector bundle of rank $j(g-j)$ with an action of $\Gamma_{j} \times \Gamma_{g-j}$ and it descends to the tensor product

$$
N^{\vee}:=\mathbb{E}_{j} \otimes \mathbb{E}_{g-j}:=p_{j}^{*}\left(\mathbb{E}_{j}\right) \otimes p_{g-j}^{*}\left(\mathbb{E}_{g-j}\right)
$$

on $\mathcal{A}_{j} \times \mathcal{A}_{g-j}$ of the pullbacks of Hodge bundles $\mathbb{E}_{j}$ and $\mathbb{E}_{g-j}$ on the factors $\mathcal{A}_{j}$ and $\mathcal{A}_{g-j}$. Here $p_{j}$ (resp. $p_{g-j}$ ) denotes the projection of $\Gamma_{j} \backslash \mathfrak{H}_{j} \times$ $\Gamma_{g-j} \backslash \mathfrak{H}_{g-j}$ onto the factor $\Gamma_{j} \backslash \mathfrak{H}_{j}$ (resp. $\Gamma_{g-j} \backslash \mathfrak{H}_{g-j}$ ). This readily can be checked by a direct computation, but also follows from the observation that the cotangent space to the moduli space $\mathcal{A}_{g}=\Gamma_{g} \backslash \mathfrak{H}_{g}$ of principally polarized complex abelian varieties at a (general) point $X^{\prime} \times X^{\prime \prime}$ with $X^{\prime}\left(\right.$ resp. $\left.X^{\prime \prime}\right)$ a principally polarized abelian variety of dimension $j$ (resp. $g-j$ ), can be identified with

$$
\operatorname{Sym}^{2}\left(T_{X^{\prime}}^{\vee}\right) \oplus\left(T_{X^{\prime}}^{\vee} \otimes T_{X^{\prime \prime}}^{\vee}\right) \oplus \operatorname{Sym}^{2}\left(T_{X^{\prime \prime}}^{\vee}\right),
$$

with $T_{X}=T_{X^{\prime}} \oplus T_{X^{\prime \prime}}$ denoting the tangent space to $X$ at the origin, and the middle term corresponds to the (co-)normal space. Since for $g=2 j$ the map 
of $\mathcal{A}_{j} \times \mathcal{A}_{j} \rightarrow \mathcal{A}_{g}$ has degree 2 , we see the development of $f$ along $\mathfrak{H}_{j} \times \mathfrak{H}_{j}$ is symmetric, that is, invariant under the interchange of factors.

The Hodge bundle $\mathbb{E}_{g}$ on $\mathcal{A}_{g}$ is associated to the standard representation of $\mathrm{GL}_{g}$. In concrete terms it corresponds to the factor of automorphy $c \tau+d$. Its determinant is denoted $L_{g}$. The bundle $N^{\vee}$ then corresponds to the tensor product of the standard representations of $\mathrm{GL}_{j}$ and $\mathrm{GL}_{g-j}$.

If $f$ is a scalar-valued modular form of weight $k$, that is, a section of $L_{g}^{k}$, we can develop $f$ in a point $\left(\tau^{\prime}, \tau^{\prime \prime}\right) \in \mathfrak{H}_{j} \times \mathfrak{H}_{g-j}$ in a Taylor series: if we write $\tau \in \mathfrak{H}_{g}$ as $\left(\begin{array}{cc}\tau^{\prime} & z \\ z^{\prime} & \tau^{\prime \prime}\end{array}\right)$ then we have

$$
f=t_{0}(f)+t_{1}(f)+t_{2}(f)+\ldots,
$$

where $t_{r}(f)$ is the sum of the terms of degree $r$ in the coordinates $z_{a, b}$ with $1 \leq a \leq j, 1 \leq b \leq g-j$ of $z$; in other words we develop $f$ along $\mathfrak{H}_{j} \times \mathfrak{H}_{g-j}$. If $t_{0}(f)$ vanishes then we can interpret the term $t_{1}(f)$ as a section of the conormal bundle tensored with $L_{g}^{k}$ over $\mathfrak{H}_{j} \times \mathfrak{H}_{g-j}$ and it descends to a section of the vector bundle $L_{j}^{k} \otimes L_{g-j}^{k} \otimes N^{\vee}$ on $\mathcal{A}_{j} \times \mathcal{A}_{g-j}$, where $L_{j}^{k} \otimes$ $L_{g-j}^{k}$ is the restriction of the line bundle $L_{g}^{k}$ on $\mathcal{A}_{g}$ whose sections are modular forms of weight $k$. More generally, if $t_{i}(f)=0$ for $i=0, \ldots, r-1$ then $t_{r}(f)$ gives a section of $L_{j}^{k} \otimes L_{g-j}^{k} \otimes \operatorname{Sym}^{r}\left(N^{\vee}\right)$. In fact, the $n$th jet bundle $B_{n}$ tensored by $L_{g}^{k}$ admits a filtration

$$
L_{g}^{k} \otimes B_{0} \subset L_{g}^{k} \otimes B_{1} \subset \ldots \subset L_{g}^{k} \otimes B_{n}
$$

and the quotient $B_{j} / B_{j-1}$ is isomorphic to $\operatorname{Sym}^{j}\left(N^{\vee}\right)$.

We consider the $r$ th symmetric power $\operatorname{Sym}^{r}\left(N^{\vee}\right)$. Note that if we have two vector spaces $V_{1}$ and $V_{2}$ of dimension $j$ and $g-j$ with the standard $\mathrm{GL}_{j}$ and $\mathrm{GL}_{g-j}$-action then we have a natural map

$$
\operatorname{Sym}^{r}\left(V_{1} \otimes V_{2}\right) \rightarrow \operatorname{Sym}^{r}\left(V_{1}\right) \otimes \operatorname{Sym}^{r}\left(V_{2}\right)
$$

which is an isomorphism if either $V_{1}$ or $V_{2}$ has dimension 1 . This happens if $j=1$ and then $\operatorname{Sym}^{r}\left(\mathbb{E}_{1} \otimes \mathbb{E}_{g-1}\right) \cong \mathbb{E}_{1}^{r} \otimes \operatorname{Sym}^{r}\left(\mathbb{E}_{g-1}\right)$. We begin by considering this case.

Proposition 2.1. Let $f \in M_{k}\left(\Gamma_{g}\right)$. If the restriction of $f$ along $\mathfrak{H}_{1} \times \mathfrak{H}_{g-1}$ vanishes to order $r$ and not to higher order, then the lowest order terms of $f$ along $\mathfrak{H}_{1} \times \mathfrak{H}_{g-1}$ define a non-vanishing element $f^{\prime} \otimes f^{\prime \prime} \in M_{k^{\prime}}\left(\Gamma_{1}\right) \otimes$ $M_{k^{\prime \prime}}\left(\Gamma_{g-1}\right)$ where the weight $k^{\prime}$ of $f^{\prime}$ equals $k+r$ and that of $f^{\prime \prime}$ equals $k^{\prime \prime}=(r, 0, \ldots, 0, k)$. 
Proof. If $t_{j}(f)=0$ for $j<r$ but $t_{r}(f) \neq 0$ then $t_{r}(f)$ defines a non-zero section of $\operatorname{Sym}^{r}\left(N^{\vee}\right) \otimes L_{\mid \mathcal{A}_{1, g-1}}^{k}$ and the pullback of this to $\mathcal{A}_{1} \times \mathcal{A}_{g-1}$ is equal to $\mathbb{E}_{1}^{r+k} \otimes \operatorname{Sym}^{r}\left(\mathbb{E}_{g-1}\right) \otimes L_{g-1}^{k}$.

For given partition $g=j+(g-j)$ of $g$ we let $\mathrm{GL}_{j} \times \mathrm{GL}_{g-j}$ be the subgroup of $\mathrm{GL}_{g}$ that respects a decomposition $V=V_{1} \oplus V_{2}$. In general, the space $\operatorname{Sym}^{k}\left(V_{1} \otimes V_{2}\right)$ admits a decomposition as a direct sum of tensor products of irreducible representations of $\mathrm{GL}_{j} \times \mathrm{GL}_{g-j}$ in which the term $\operatorname{Sym}^{k}\left(V_{1}\right) \otimes \operatorname{Sym}^{k}\left(V_{2}\right)$ has high codimension.

If $f$ is a scalar-valued Siegel modular form of weight $k$ vanishing up to order $r$ along $\mathfrak{H}_{j} \times \mathfrak{H}_{g-j}$ then we look at its term of order $r$. This defines a section of

$$
L_{j}^{k} \otimes L_{g-j}^{k} \otimes \operatorname{Sym}^{r}\left(\mathbb{E}_{j} \otimes \mathbb{E}_{g-j}\right)
$$

and we can project it to each of the irreducible constituents of this bundle; in particular we can consider the projection of the second factor to $\operatorname{Sym}^{r}\left(\mathbb{E}_{j}\right) \otimes$ $\operatorname{Sym}^{r}\left(\mathbb{E}_{g-j}\right)$. The following proposition describes the result.

Proposition 2.2. If $f \in M_{k}\left(\Gamma_{g}\right)$ vanishes up to order $r$ along $\mathfrak{H}_{j} \times \mathfrak{H}_{g-j}$ then the projection of its rth term to $L_{j}^{k} \otimes L_{g-j}^{k} \otimes \operatorname{Sym}^{r}\left(\mathbb{E}_{j}\right) \otimes \operatorname{Sym}^{r}\left(\mathbb{E}_{g-j}\right)$ gives a tensor product $f^{\prime} \otimes f^{\prime \prime}$ with $f^{\prime} \in M_{k^{\prime}}\left(\Gamma_{j}\right)$ (resp. $f^{\prime \prime} \in M_{k^{\prime \prime}}\left(\Gamma_{g-j}\right)$ ) with $k^{\prime}$ (resp. $\left.k^{\prime \prime}\right)$ a vector of length $j($ resp. $g-j)$ of the form $(r, 0, \ldots, 0, k)$

In general, if $V_{n}$ is the standard representation of $\mathrm{GL}_{n}$ and we write it as $V_{n}=V_{j} \oplus V_{g-j}$ then we have a decomposition of $\operatorname{Sym}^{r}\left(V_{j} \otimes V_{g-j}\right)$ as a direct sum of isotypic subspaces for $\mathrm{GL}_{j} \times \mathrm{GL}_{g-j}$

$$
\operatorname{Sym}^{r}(V)=\oplus m_{\lambda^{\prime}, \lambda^{\prime \prime}} W_{\lambda^{\prime}} \otimes W_{\lambda^{\prime \prime}}
$$

If the restriction of a scalar-valued Siegel modular form $f$ vanishes up to order $r$ along $\mathfrak{H}_{j} \times \mathfrak{H}_{g-j}$, then the projection onto any of the isotypic spaces in (2) gives a vector-valued modular form.

But we can also find modular forms further on in the Taylor expansion. The following proposition gives an example.

We write a form $f \in M_{k}\left(\Gamma_{g}\right)$ as a Taylor expansion $f=\sum_{n>0} t_{r}(f)$ and decompose each term in its isotypic components $t_{n}(f)=\sum_{\lambda} t_{n, \lambda}(f)$, where $\lambda$ indexes the irreducible representations $R_{\lambda}$ that occur in $\operatorname{Sym}^{n}\left(V_{j} \otimes V_{g-j}\right)$.

Proposition 2.3. Let $f \in M_{k}\left(\Gamma_{g}\right)$ vanish to order $r$ on $\mathfrak{H}_{j} \times \mathfrak{H}_{g-j}$ and write $f=\sum_{n \geq r} t_{r}(f)$. Let $R_{\alpha}$ be an irreducible representation of $\mathrm{GL}_{j} \times$ 
$\mathrm{GL}_{g-j}$ occurring in the representation $\operatorname{Sym}^{r+1}\left(V_{j} \otimes V_{g-j}\right)$ that does not occur in $\left(V_{j} \otimes V_{g-j}\right) \otimes R_{\beta}$ for any $R_{\beta}$ for which $t_{r, \beta}(f) \neq 0$. Then the projection $t_{r+1, \alpha}(f)$ of $f$ to the $\alpha$-isotypic component of the $(r+1)$ th step is a modular form.

Proof. Exterior differentiation $d$ induces the homogeneous differential operator on $\mathfrak{H}_{j} \times \mathfrak{H}_{g-j}$ from $\operatorname{Sym}^{r}\left(N^{\vee}\right)$ to $\operatorname{Sym}^{r+1}\left(N^{\vee}\right)$. In terms of representations of GL it corresponds to multiplication by $V_{j} \otimes V_{g-j}$. In fact, the functional equation for $f$ says that $f(\gamma \tau)=\rho(\gamma, \tau) f(\tau)$ for $\gamma \in \Gamma_{j} \times \Gamma_{g-j} \subset \Gamma_{g}$ and this implies that $t_{r}(f(\gamma \tau))+t_{r+1}(f(\gamma \tau))$ equals

$$
t_{0}(\rho(\gamma, \tau)) t_{r}(f(\tau))+t_{1}(\rho(\gamma, \tau)) t_{r}(f(\tau))+t_{0}(\rho(\gamma, \tau)) t_{r+1}(f(\tau))
$$

and since $t_{r}(f)$ is a scalar-valued modular form we see that

$$
t_{r+1}(f(\gamma \tau))=t_{1}(\rho(\gamma, \tau)) t_{r}(f(\tau))+t_{0}(\rho(\gamma, \tau)) t_{r+1}(f(\tau))
$$

Note that $t_{1}(\rho(\gamma, \tau))$ is isomorphic to $V_{j} \otimes V_{g-j}$ as a GL-representation. Projection on the isotypic terms gives the result.

Question 2.4. In connection with the method presented here it is an interesting question what the ideal is of scalar-valued Siegel modular forms of degree $g$ vanishing on $\mathfrak{H}_{j} \times \mathfrak{H}_{g-j}$. For $g=2$ and $j=1$ the answer is the ideal generated by $\chi_{10}$, but for other cases, even for $g=3$, the answer seems unknown. See also Igusa [12] and Sasaki [19].

\section{Example: Restricting The Schottky Form}

We illustrate the method by the example of the restriction of the Schottky form $J_{8}$, a cusp form of weight 8 in degree 4 along degree $2+2$ and degree $3+1$, cf. [11]. Its divisor in $\mathcal{A}_{4}$ is the closure of the locus of Jacobians. The Schottky form can be described in various ways: as the difference of the theta series attached to the two unimodular lattices $E_{8} \oplus E_{8}$ and $D_{16}^{+}$, but also as the Ikeda lift of $\Delta \in S_{12}\left(\Gamma_{1}\right)$. For yet other descriptions, we refer to [18] top of the page 209. Since there are no cusp forms of weight 8 on $\Gamma_{2}$, its restriction to $\mathfrak{H}_{2} \times \mathfrak{H}_{2}$ vanishes. Using the action of the matrix

$$
\left(\begin{array}{ll}
a & 0 \\
0 & d
\end{array}\right) \quad \text { with } a=d=\left(\begin{array}{cc}
1_{2} & 0 \\
0 & -1_{2}
\end{array}\right)
$$

we see that $t_{i}\left(J_{8}\right)$ in the development (1) vanishes for $i$ odd. If $V=V^{\prime} \oplus V^{\prime \prime}$ is the standard representation of $\mathrm{GL}_{4}$ and $V^{\prime}$ and $V^{\prime \prime}$ are the standard 
representations of $\mathrm{GL}_{2}$ then we have a decomposition

$$
\operatorname{Sym}^{2}\left(V^{\prime} \otimes V^{\prime \prime}\right) \cong \operatorname{Sym}^{2}\left(V^{\prime}\right) \otimes \operatorname{Sym}^{2}\left(V^{\prime \prime}\right) \oplus \wedge^{2} V^{\prime} \otimes \wedge^{2} V^{\prime \prime},
$$

so starting with a modular form $f$ of weight $k$ that vanishes with order 2 along $\mathfrak{H}_{2} \times \mathfrak{H}_{2}$ the term $t_{2}(f)$ gives modular forms in

$$
M_{2, k}\left(\Gamma_{2}\right) \otimes M_{2, k}\left(\Gamma_{2}\right) \quad \text { and } \quad M_{0, k+1}\left(\Gamma_{2}\right) \otimes M_{0, k+1}\left(\Gamma_{2}\right)
$$

But in the case at hand $k=8$ and the spaces $S_{2,8}\left(\Gamma_{2}\right)$ and $S_{0,9}\left(\Gamma_{2}\right)$ vanish. Therefore we pass to order 4 . Here we have the identification of $\operatorname{Sym}^{4}\left(V^{\prime} \otimes\right.$ $\left.V^{\prime \prime}\right)$ with

$$
\begin{array}{r}
\operatorname{Sym}^{4}\left(V^{\prime}\right) \otimes \operatorname{Sym}^{4}\left(V^{\prime \prime}\right) \bigoplus\left(\operatorname{Sym}^{2}\left(V^{\prime}\right) \otimes \wedge^{2}\left(V^{\prime}\right)\right) \otimes\left(\operatorname{Sym}^{2}\left(V^{\prime \prime}\right) \otimes \wedge^{2}\left(V^{\prime \prime}\right)\right) \\
\bigoplus\left(\wedge^{2}\left(V^{\prime}\right) \otimes \wedge^{2}\left(V^{\prime}\right)\right) \otimes\left(\wedge^{2}\left(V^{\prime \prime}\right) \otimes \wedge^{2}\left(V^{\prime \prime}\right)\right) .
\end{array}
$$

Thus for a modular form of weight $k$ on $\Gamma_{4}$ which vanishes at order four along $\mathfrak{H}_{2} \times \mathfrak{H}_{2}, t_{4}(f)$ lies in

$$
\operatorname{Sym}^{2} M_{4, k}\left(\Gamma_{2}\right) \oplus \operatorname{Sym}^{2} M_{2, k+1}\left(\Gamma_{2}\right) \oplus \operatorname{Sym}^{2} M_{0, k+2}\left(\Gamma_{2}\right)
$$

The following Proposition shows that $\Delta$ and its siblings of degree 2 and 3 occur in the development of the Schottky form.

Proposition 3.1. For the Schottky form $J_{8} \in S_{8}\left(\Gamma_{4}\right)$ of weight 8 on $\Gamma_{4}$ the term $t_{4}\left(J_{8}\right)$ in the restriction from degree 4 to degree $2+2$ is a non-zero multiple of

$$
\chi_{10} \otimes \chi_{10} \in \operatorname{Sym}^{2} S_{10}\left(\Gamma_{2}\right)
$$

with $\chi_{10}$ a generator of $S_{10}\left(\Gamma_{2}\right)$, while the projection of $t_{6}\left(J_{8}\right)$ to $\operatorname{Sym}^{2} S_{6,8}\left(\Gamma_{2}\right)$ is equal to a non-zero multiple of

$$
\chi_{6,8} \otimes \chi_{6,8} \in \operatorname{Sym}^{2} S_{6,8}\left(\Gamma_{2}\right)
$$

where $\chi_{6,8}$ is a generator of $S_{6,8}\left(\Gamma_{2}\right)$. The term $t_{4}\left(J_{8}\right)$ in the restriction from degree 4 to degree $3+1$ is a non-zero multiple of

$$
\chi_{4,0,8} \otimes \Delta
$$

with $\chi_{4,0,8} \in S_{4,0,8}\left(\Gamma_{3}\right)$ a generator. 
Proof. Since $S_{4,8}\left(\Gamma_{2}\right)=S_{2,9}\left(\Gamma_{2}\right)=(0) \quad$ we see that $t_{4}\left(J_{8}\right)$ lies in $\operatorname{Sym}^{2} S_{10}\left(\Gamma_{2}\right)$. By calculating the coefficients one sees that it does not vanish. Indeed, if $\left(e_{1}, e_{2}\right)$ is a basis of $V_{1}$ and $\left(f_{1}, f_{2}\right)$ is a basis of $V_{2}$ we let $e_{i} \otimes f_{j}$ correspond to $\tau_{i j}$. The projection of $\operatorname{Sym}^{4}\left(V_{1} \otimes V_{2}\right)$ on the direct summand $\left(\wedge^{2} V_{1} \otimes \wedge^{2} V_{1}\right) \otimes\left(\wedge^{2} V_{2} \otimes \wedge^{2} V_{2}\right)$ is then given by

$$
\frac{\partial^{4}}{\partial \tau_{13}^{2} \partial \tau_{24}^{2}}+\frac{\partial^{4}}{\partial \tau_{14}^{2} \partial \tau_{23}^{2}}-2 \frac{\partial^{4}}{\partial \tau_{13} \partial \tau_{14} \partial \tau_{23} \partial \tau_{24}}
$$

and a direct computation shows that

$$
t_{4}\left(J_{8}\right)=12 \chi_{10} \otimes \chi_{10} .
$$

Playing the same game with the term $t_{6}\left(J_{8}\right)$ we find components in

$$
\operatorname{Sym}^{6}(V)=\sum_{\lambda} V_{\lambda}^{\prime} \otimes V_{\lambda}^{\prime \prime}
$$

where $\lambda$ runs through $(6,0),(4,1),(2,2)$ and $(0,3)$. By Proposition 2.3 all components except the last one can give modular forms. But the spaces $S_{4,9}\left(\Gamma_{2}\right), S_{2,10}\left(\Gamma_{2}\right)$ and $S_{0,11}\left(\Gamma_{2}\right)$ are zero, therefore we find

$$
t_{6}\left(J_{8}\right) \in \operatorname{Sym}^{2} S_{6,8}\left(\Gamma_{2}\right)
$$

and a calculation shows that this term does not vanish. The argument for the restriction from degree 4 to $3+1$ is similar.

More details on the forms $\chi_{6,8}$ and the form in $S_{4,0,8}\left(\Gamma_{3}\right)$ can be found in sections 8 and 9 .

\section{Dimensions of Spaces of Scalar Cusp Forms}

It will be useful to have a table for the dimensions of scalar-valued Siegel modular cusp forms of weight $4 \leq k \leq 18$ and degree $1 \leq g \leq 8$. We will use this table as a heuristic tool that tells us where to look for modular forms vanishing on $\mathfrak{H}_{j} \times \mathfrak{H}_{g-j}$. The tables of Taïbi [20] provide the dimensions in many cases, though some of his results are conditional. For $g=2$ the dimensions were determined by Igusa, for $g=3$ by Tsuyumine. For degree 4 and some higher degrees there are results of Poor and Yuen in [18]. 


\begin{tabular}{|c|r|l|l|r|r|r|r|r|r|r|r|r|}
\hline$g \backslash k$ & $<8$ & 8 & 9 & 10 & 11 & 12 & 13 & 14 & 15 & 16 & 17 & 18 \\
\hline 1 & 0 & 0 & 0 & 0 & 0 & 1 & 0 & 0 & 0 & 1 & 0 & 1 \\
2 & 0 & 0 & 0 & 1 & 0 & 1 & 0 & 1 & 0 & 2 & 0 & 2 \\
3 & 0 & 0 & 0 & 0 & 0 & 1 & 0 & 1 & 0 & 3 & 0 & 4 \\
4 & 0 & 1 & 0 & 1 & 0 & 2 & 0 & 3 & 0 & 7 & 0 & 12 \\
5 & 0 & 0 & 0 & 0 & 0 & 2 & 0 & 3 & 0 & 13 & 0 & 28 \\
6 & 0 & 0 & 0 & 1 & 0 & 3 & $?$ & 9 & 0 & 33 & 0 & 117 \\
7 & 0 & 0 & 0 & 0 & 0 & 3 & 0 & 9 & 0 & 83 & 0 & $?$ \\
8 & 0 & $?$ & 0 & $\geq 1$ & 0 & $\geq 4$ & $\geq 1$ & $\geq 23$ & $\geq 2$ & $\geq 234$ & $?$ & $?$ \\
\hline
\end{tabular}

\section{Restricting from Degree Three}

Restriction of forms on $\Gamma_{2}$ to forms on $\Gamma_{1} \times \Gamma_{1}$ does not produce vectorvalued modular forms, just scalar-valued ones, though it shows how much the siblings of Delta are related: $\chi_{10} \in S_{10}\left(\Gamma_{2}\right)$ vanishes on $\mathfrak{H}_{1} \times \mathfrak{H}_{1}$ and its first non-vanishing term, $t_{2}$, gives $\Delta \otimes \Delta$, while in a similar way Igusa's cusp form $\chi_{35} \in S_{35}\left(\Gamma_{2}\right)$ produces $\Delta^{2} e_{12} \otimes \Delta^{2} e_{12}$ with $e_{12}$ the Eisenstein series of weight 12 on $\Gamma_{1}$.

The rings of scalar-valued Siegel modular forms on $\Gamma_{g}$ for $g \leq 3$ are well-known. $([9,21])$. A generator $\psi_{12}$ of $S_{12}\left(\Gamma_{3}\right)$ is given by the following combination of theta series associated to Niemeier lattices (of rank 24)

$$
\psi_{12}=\frac{1}{1152}\left(-\vartheta_{\text {Leech }}+6 \vartheta_{24 A_{1}}-8 \vartheta_{12 A_{2}}+3 \vartheta_{3 A_{8}}\right),
$$

where we refer for example to [16] for notations for lattices. The coefficients in the Fourier expansion $\sum_{N>0} a(N) e^{2 \pi i \operatorname{Tr}(N \tau)}$ of $\psi_{12}$ that we need are $\left.a\left(1_{3}\right)=164, a\left(A_{1}(1 / 2)\right) \oplus A_{2}(1 / 2)\right)=18$ and $a\left(A_{3}(1 / 2)\right)=1$. Thus the restriction of $\psi_{12}$ to $\mathfrak{H}_{1} \times \mathfrak{H}_{2}$ does not vanish and equals $24 \Delta \otimes \chi_{12}$.

The restriction of a cusp form in $S_{14}\left(\Gamma_{3}\right)$ to $\mathfrak{H}_{1} \times \mathfrak{H}_{2}$ must vanish. Let $F$ be the cusp form of weight 14 that generates $S_{14}\left(\Gamma_{3}\right)$. The form $F$ was constructed first by Miyawaki ([15, p. 314-315]) and later by Ikeda as a lift ([13]). We recall its construction. Let

$$
D_{16}^{+}=\left\{x \in \mathbb{Q}^{16} \mid 2 x_{i} \in \mathbb{Z}, x_{i}-x_{j} \in \mathbb{Z}, x_{1}+\ldots+x_{16} \in 2 \mathbb{Z}\right\}
$$

be the unimodular lattice of rank 16 which is not $E_{8} \oplus E_{8}$. Let $Q$ be the $3 \times 16$ complex matrix $\left(1_{3}, \rho 1_{3}, \rho^{2} 1_{3}, 0\right) \in \operatorname{Mat}(3 \times 16, \mathbb{C})$, with $\rho=e^{2 \pi i / 3}$. Then for a triple $\left(v_{1}, v_{2}, v_{3}\right)$ of vectors from $D_{16}^{+}$we get a $3 \times 3$ matrix $Q\left(v_{1}, v_{2}, v_{3}\right)$ and we define $F$ by

$$
F(\tau)=\sum_{v_{1}, v_{2}, v_{3} \in D_{16}^{+}} \operatorname{Re}\left(\operatorname{det}\left(Q\left(v_{1}, v_{2}, v_{3}\right)\right)^{6}\right) e^{\pi i \sum_{i, j=1}^{3}\left(v_{i}, v_{j}\right) \tau_{i j}}
$$


for $\tau=\left(\tau_{i j}\right) \in \mathfrak{H}_{3}$.

We shall denote the Eisenstein series of weight $k$ for $\Gamma_{1}$ by $e_{k}$ and for $\Gamma_{g}$ with $g \geq 2$ by $E_{k}$.

Proposition 5.1. The lowest non-zero term in the development of $F$ along $\mathfrak{H}_{2} \otimes \mathfrak{H}_{1}$ is $t_{2}(F)$ and it is a non-zero multiple of the form $\chi_{2,14} \otimes \Delta e_{4}$, where $\Delta e_{4} \in S_{16}\left(\Gamma_{1}\right)$ and $\chi_{2,14}=\left[\chi_{10}, E_{4}\right] \in S_{2,14}\left(\Gamma_{2}\right)$.

Proof. We claim that the term $t_{2}(F)$ starts as follows

$$
t_{2}(F)=(2 \pi i)^{2}\left(\begin{array}{c}
4 q_{12}^{-1}+4+2 x+4 q_{12} \\
4\left(q_{12}-q_{12}^{-1}\right) \\
4 q_{12}^{-1}+4+2 x+4 q_{12}
\end{array}\right) q_{1} q_{2} q_{3}+\cdots
$$

where $x$ is the Fourier coefficient of $\left(\begin{array}{ccc}1 & 0 & 0 \\ 0 & 1 & 1 / 2 \\ 0 & 1 / 2 & 1\end{array}\right)$ and where $q_{j}=e^{2 \pi i \tau_{j}}$ for $j=1,2,3$ and $q_{12}=e^{2 \pi i \tau_{12}}$. This can be deduced from [15] (last table there) and the fact that the Fourier coefficients $a(N)$ of $F$ satisfy $a(N)=a\left(U^{t} N U\right)$ for $U \in \mathrm{GL}(3, \mathbb{Z})$. We know that $t_{2}(F)$ lies in $S_{2,14}\left(\Gamma_{2}\right) \otimes S_{16}\left(\Gamma_{1}\right)$ and does not vanish. We can construct a generator of $S_{2,14}\left(\Gamma_{2}\right)$ by the bracket construction, cf. [7, 10]. The Fourier expansions of $\chi_{10}$ and the Eisenstein series $E_{4} \in M_{4}\left(\Gamma_{2}\right)$ start as follows:

$$
\chi_{10}(\tau)=\left(q_{12}^{-1}-2+q_{12}\right) q_{1} q_{2}+\cdots \quad \text { and } \quad E_{4}(\tau)=1+240\left(q_{1}+q_{2}\right)+\cdots
$$

so we have

$$
\left[\chi_{10}, E_{4}\right]=10\left(\begin{array}{c}
q_{12}^{-1}-2+q_{12} \\
q_{12}-q_{12}^{-1} \\
q_{12}^{-1}-2+q_{12}
\end{array}\right) q_{1} q_{2}+\cdots
$$

The Fourier expansion of $\Delta e_{4}$ starts by $\Delta e_{4}\left(\tau_{3}\right)=q_{3}+\ldots$ so we get

$$
\left[\chi_{10}, E_{4}\right]\left(\begin{array}{cc}
\tau_{11} & \tau_{12} \\
\tau_{12} & \tau_{2}
\end{array}\right) \otimes \Delta e_{4}\left(\tau_{3}\right)=10\left(\begin{array}{c}
q_{12}^{-1}-2+q_{12} \\
q_{12}-q_{12}^{-1} \\
q_{12}^{-1}-2+q_{12}
\end{array}\right) q_{1} q_{2} q_{3}+\ldots
$$

It follows that $x=-6=a\left(\left(\begin{array}{ccc}1 & 0 & 0 \\ 0 & 1 & 1 / 2 \\ 0 & 1 / 2 & 1\end{array}\right)\right)$ for the unknown Fourier coefficient of $F$.

In weight 18 there is a well-known scalar-valued cusp form $\chi_{18}$ of degree 3 that vanishes along the locus of Jacobians of hyperelliptic curves of degree 3 . It is defined as the product of the 36 even theta characteristics in degree 3 . 
The Fourier expansion of this $\chi_{18}$ starts as follows

$$
2^{28}\left(108-60\left(q_{12}^{-1}+q_{12}+q_{13}^{-1}+q_{13}+q_{23}^{-1}+q_{23}+\cdots\right) q_{1}^{2} q_{2}^{2} q_{3}^{2}+\cdots\right)
$$

showing that $\chi_{18}$ vanishes of order 2 at infinity. The restriction to $\mathfrak{H}_{2} \times \mathfrak{H}_{1}$ vanishes because this restriction lies in $S_{18}\left(\Gamma_{2}\right) \otimes S_{18}\left(\Gamma_{1}\right)$, and because $\chi_{18}$ vanishes twice at infinity the components of its restriction do so too, and there is no cusp form of weight $<24$ on $\Gamma_{1}$ vanishing twice at infinity.

Proposition 5.2. Along $\mathfrak{H}_{2} \times \mathfrak{H}_{1}$ we have

$$
t_{6}\left(\chi_{18}\right)=c \chi_{10} \chi_{6,8} \otimes \Delta^{2} \in S_{6,18}\left(\Gamma_{2}\right) \otimes S_{24}\left(\Gamma_{1}\right),
$$

where $c \neq 0$ and $\chi_{6,8}$ is a generator of $S_{6,8}\left(\Gamma_{2}\right)$.

Proof. From the fact that there is no cusp form of weight less than 24 on $\Gamma_{1}$ that vanishes twice at the cusp it follows that $t_{i}\left(\chi_{18}\right)$ vanishes for $i \leq 5$. We know that the $t_{6}\left(\chi_{18}\right)$ lies in $S_{6,18}\left(\Gamma_{2}\right) \otimes S_{24}\left(\Gamma_{1}\right)$. But the subspace of $S_{24}\left(\Gamma_{1}\right)$ of elements vanishing twice at infinity is generated by $\Delta^{2}$. Moreover, the calculation

$$
\left(\begin{array}{c}
\frac{\partial^{6} \chi_{18}}{\partial \tau_{13}^{1}} \\
6 \frac{\partial^{6} x_{18}}{\partial \tau_{13}^{5} \partial \tau_{23}} \\
\vdots \\
6 \frac{\partial^{6} x_{18}}{\partial \tau_{13} \partial \tau_{23}^{5}} \\
\frac{\sigma^{6} \chi_{18}}{\partial \tau_{23}^{6}}
\end{array}\right)\left(\begin{array}{ccc}
\tau_{1} & \tau_{12} & 0 \\
\tau_{12} & \tau_{2} & 0 \\
0 & 0 & \tau_{3}
\end{array}\right)=\left(\begin{array}{c}
0 \\
0 \\
0 \\
q_{12}^{-2}-4 q_{12}^{-1}+6-4 q_{12}+q_{12}^{2} \\
-2 q_{12}^{-2}+4 q_{12}^{-1}-4 q_{12}+2 q_{12}^{2} \\
q_{12}^{-2}-4 q_{12}^{-1}+6-4 q_{12}+q_{12}^{2} \\
0 \\
0
\end{array}\right) q_{1}^{2} q_{2}^{2} q_{3}^{2}+\ldots
$$

shows that it does not vanish and is divisible by $\chi_{10}$ because substitution of $q_{12}=1$ gives zero. Since $\operatorname{dim} S_{6,8}\left(\Gamma_{2}\right)=1$ the result follows.

\section{Restricting from Degree 4}

We begin by listing the modular forms that we are going to restrict. As before, we denote by $e_{k}$ the Eisenstein series of weight $k$ on $\Gamma_{1}$ and by $E_{k}$ the Eisenstein series of weight $k$ in higher genera, always normalized such that their Fourier expansion starts with 1.

Besides the Schottky form $J_{8}$ that generates $S_{8}\left(\Gamma_{4}\right)$ we have the generator $F_{10}=-I_{4}\left(e_{4} \Delta\right) / 168 \in S_{10}\left(\Gamma_{4}\right)$ where $I_{4}$ is the Ikeda lift $I_{4}: S_{16}\left(\Gamma_{1}\right) \rightarrow$ $S_{10}\left(\Gamma_{4}\right)$, the two generators $G_{1}=I_{4}\left(e_{4}^{2} \Delta\right) / 360$ and $G_{2}=-J_{8} E_{4} / 2$ of $S_{12}\left(\Gamma_{4}\right)$, the three Hecke eigenforms $H_{1}, H_{2}, H_{3}$ that generate $S_{14}\left(\Gamma_{4}\right)$, see 
[18, p. 213] (but note that the eigenvalues given there are not correct; in fact, the expressions of $f_{7}$ and $f_{8}$ on page 214 and the eigenvalues for $J_{8}$ in table 2 on page 218 are incorrect), where

$$
H_{1}=I_{4}\left(\Delta e_{4}^{3}-(156-12 \alpha) \Delta^{2}\right), \quad \text { and } \quad H_{2}=I_{4}\left(\Delta e_{4}^{3}-(156+12 \alpha) \Delta^{2}\right)
$$

with $\alpha=\sqrt{144169}$. One can calculate the first Fourier coefficients. We give the results in a table. The coefficients of $H_{2}$ are the conjugates of those of $H_{1}$. The space $S_{14}\left(\Gamma_{4}\right)$ contains $E_{6} J_{8}$.

Note that the paper 14] gives a closed formula for the eigenvalues of an Ikeda lift.

\begin{tabular}{|r|r|r|r|r|r|r|}
\hline & $J_{8}$ & $F_{10}$ & $G_{1}$ & $G_{2}$ & $H_{1}$ & $H_{3}$ \\
\hline $1_{4}$ & 40 & 472 & -4440 & -40 & $-434984-968 \alpha$ & -2080 \\
$2 A_{1}(1 / 2) \oplus A_{2}(1 / 2)$ & -12 & -36 & -492 & 12 & $63132+204 \alpha$ & 288 \\
$2 A_{2}(1 / 2)$ & 6 & 72 & -78 & -6 & $-44904-48 \alpha$ & -198 \\
$A_{1}(1 / 2) \oplus A_{3}(1 / 2)$ & 2 & -22 & -38 & -2 & $4994-22 \alpha$ & 28 \\
$A_{4}(1 / 2)$ & -1 & 2 & 1 & 1 & $-274+2 \alpha$ & -5 \\
$D_{4}(1 / 2)$ & 1 & 1 & -3 & -1 & $-467+\alpha$ & 2 \\
\hline
\end{tabular}

According to [3] we should have $\operatorname{dim} S_{4,0,8}\left(\Gamma_{3}\right)=1, \operatorname{dim} S_{2,0,10}\left(\Gamma_{3}\right)=1$ and $\operatorname{dim} S_{2,0,14}\left(\Gamma_{3}\right)=2$. We denote the generating eigenforms by $\chi_{4,0,8}$, $\chi_{2,0,10}$, and $\chi_{2,0,14}$ and $\chi_{2,0,14}^{\prime}$. The form $\chi_{2,0,14}$ and its conjugate $\chi_{2,0,14}^{\prime}$ are lifts with Hecke eigenvalues of the form

$$
a(p)\left(p^{11}+b(p)+p^{12}\right)
$$

with $a(p)$ the eigenvalue of the eigenform of $S_{16}\left(\Gamma_{1}\right)$ and $b(p)$ the eigenvalue of a Hecke eigenform in $S_{24}\left(\Gamma_{1}\right)$.

Proposition 6.1. By restricting scalar-valued modular cusp forms of degree 4 and small weight to $\mathfrak{H}_{3} \times \mathfrak{H}_{1}$ we find (a non-zero multiple of) the vector-valued modular forms as in the table below where $\chi_{0,0,12}=\psi_{12}$.

\begin{tabular}{|r|r|r|r|r|}
\hline$k$ & $\operatorname{dim} S_{k}\left(\Gamma_{4}\right)$ & \multicolumn{1}{|c|}{ form } & $r$ & $t_{r}$ \\
\hline 8 & 1 & $J_{8}$ & 4 & $\chi_{4,0,8} \otimes \Delta$ \\
10 & 1 & $F_{10}$ & 2 & $\chi_{2,0,10} \otimes \Delta$ \\
12 & 2 & $G_{1}$ & 0 & $\chi_{0,0,12} \otimes \Delta$ \\
& & $G_{2}$ & 2 & $E_{4} \chi_{6,8} \otimes e_{4} \Delta$ \\
14 & 3 & $H_{1}$ & 2 & $\chi_{2,0,14} \otimes e_{4} \Delta$ \\
& & $H_{2}$ & 2 & $\chi_{2,0,14}^{\prime} \otimes e_{4} \Delta$ \\
& & $E_{6} J_{8}$ & 4 & $E_{6} \chi_{4,0,8} \otimes e_{6} \Delta$ \\
\hline
\end{tabular}


Proposition 6.2. By restricting scalar-valued modular cusp forms of degree 4 and small weight to $\mathfrak{H}_{2} \times \mathfrak{H}_{2}$ we find the vector-valued modular forms as in the table below.

\begin{tabular}{|r|r|r|r|r|}
\hline$k$ & $\operatorname{dim} S_{k}\left(\Gamma_{4}\right)$ & form & $r$ & $t_{r}$ \\
\hline 8 & 1 & $J_{8}$ & 6 & $\chi_{6,8} \otimes \chi_{6,8}$ \\
10 & 1 & $F_{10}$ & 0 & $\chi_{10} \otimes \chi_{10}$ \\
12 & 2 & $G_{1}$ & 0 & $\chi_{12} \otimes \chi_{12}$ \\
& & $G_{2}$ & 6 & $E_{4} \chi_{6,8} \otimes E_{4} \chi_{6,8}$ \\
14 & 3 & $H_{1}$ & 0 & $E_{4} \chi_{10} \otimes E_{4} \chi_{10}$ \\
& & $H_{1}-H_{2}$ & 2 & $\chi_{2,14} \otimes \chi_{2,14}$ \\
& & $E_{6} J_{8}$ & 6 & $E_{6} \chi_{6,8} \otimes E_{6} \chi_{6,8}+E_{6} \chi_{10} \otimes E_{6} \chi_{10}$ \\
\hline
\end{tabular}

Note that if $f$ is a form in $S_{k}\left(\Gamma_{4}\right)$ such that along $\mathfrak{H}_{2} \times \mathfrak{H}_{2}$ we have that $t_{r}(f)=0$ for $r<6$ then $t_{6}(f)$ lies in

$\operatorname{Sym}^{2}\left(S_{6, k}\left(\Gamma_{2}\right)\right) \oplus \operatorname{Sym}^{2}\left(S_{4, k+1}\left(\Gamma_{2}\right)\right) \oplus \operatorname{Sym}^{2}\left(S_{2, k+2}\left(\Gamma_{2}\right)\right) \oplus \operatorname{Sym}^{2}\left(S_{0, k+3}\left(\Gamma_{2}\right)\right)$

We can deduce the Fourier expansion of these forms. For example, the Fourier expansion of $\chi_{2,0,10} / 12(2 \pi i)^{2}$ starts by $q_{1} q_{2} q_{3}$ times

$$
\left[\left(\begin{array}{c}
-20 \\
0 \\
0 \\
-20 \\
0 \\
-20
\end{array}\right)+\left(\begin{array}{c}
-6 \\
-6 \\
0 \\
-6 \\
0 \\
14
\end{array}\right) q_{12}+\left(\begin{array}{c}
-6 \\
6 \\
0 \\
-6 \\
0 \\
14
\end{array}\right) q_{12}^{-1}+\left(\begin{array}{c}
-6 \\
0 \\
-6 \\
14 \\
0 \\
-6
\end{array}\right) q_{13}+\ldots+\left(\begin{array}{l}
1 \\
1 \\
1 \\
1 \\
0 \\
1
\end{array}\right) q_{12} q_{13}+\ldots\right]
$$

and that of $\left.\chi_{2,0,14} / 12(2 \pi i)^{2}\right)$ starts by $q_{1} q_{2} q_{3}$ times

$$
\left[\left(\begin{array}{c}
13540+20 \alpha \\
0 \\
0 \\
13540+20 \alpha \\
0 \\
13540+20 \alpha
\end{array}\right)+\left(\begin{array}{c}
-6 \alpha+1482 \\
-6 \alpha+1482 \\
0 \\
-6 \alpha+1482 \\
-6 \alpha-7758
\end{array}\right) q_{12}+\ldots+\left(\begin{array}{c}
\alpha-247 \\
\alpha-247 \\
\alpha-247 \\
\alpha-247 \\
0 \\
\alpha-247
\end{array}\right) q_{12} q_{13}+\ldots\right]
$$

\section{Restriction from Degree Six and Eight}

We begin by constructing a form of weight 10 both in degree 8 and degree 6. The Ikeda lift of $\Delta \in S_{12}\left(\Gamma_{1}\right)$ to degree 8 gives a form $I_{8}(\Delta)$ in $S_{10}\left(\Gamma_{8}\right)$. For our purpose we need a number of Fourier coefficients of $I_{8}(\Delta)=\sum_{T} b(T) e^{2 \pi i \operatorname{tr}(T \cdot \tau)}$; in fact, we need these for all positive definite half-integral symmetric matrices with diagonal equal to $(1, \ldots, 1)$. For a positive definite half-integral symmetric matrix $T$ with fundamental discriminant $D_{T}$ the Fourier coefficient is given by $b(T)=c\left(\left|D_{T}\right|\right)=c(\operatorname{det}(2 T))$, 
where

$$
h=\sum_{n \geq 1, n \equiv 0,1 \bmod 4} c(n) q^{n} \in S_{13 / 2}^{+}\left(\Gamma_{0}(4)\right)
$$

is the form of half-integral weight corresponding to $\Delta$ under the Shimura correspondence. By restricting this form $I_{8}(\Delta)$ to $\mathfrak{H}_{7} \times \mathfrak{H}_{1}$ we find linear relations between the Fourier coefficients and this gives a way of calculating further Fourier coefficients. Indeed, the restriction $r_{7,1}\left(I_{8}(\Delta)\right)$ is zero in view of $\operatorname{dim} S_{10}\left(\Gamma_{7}\right)=\operatorname{dim} S_{10}\left(\Gamma_{1}\right)=0$ and we thus find that the Fourier coefficient at $\left.N \otimes A_{1}(1 / 2)\right)$ of $r_{7,1}\left(I_{8}(\Delta)\right)$ is given by

$$
\sum_{N \oplus A_{1}(1 / 2)=T} b\left(I_{8}(\Delta), T\right),
$$

where the sum runs over all positive definite $T$ with upper left block $N$ and lower right block $A_{1}(1 / 2)$. This gives a relation $b\left(I_{8}(\Delta), A_{7}(1 / 2) \oplus\right.$ $\left.A_{1}(1 / 2)\right)+56 b\left(I_{8}(\Delta), E_{8}(1 / 2)\right)=0$. In this way we obtain a number of relations between the Fourier coefficients.

Furthermore we consider the restriction of $I_{8}(\Delta)$ to $\mathfrak{H}_{6} \times \mathfrak{H}_{2}$. We know that $S_{10}\left(\Gamma_{2}\right)$ is generated by the form $\chi_{10}$ and that $\operatorname{dim} S_{10}\left(\Gamma_{6}\right)=1$. Then restricting further to $\mathfrak{H}_{5} \times \mathfrak{H}_{1} \times \mathfrak{H}_{2}, \mathfrak{H}_{4} \times \mathfrak{H}_{2} \times \mathfrak{H}_{2}$ and $\mathfrak{H}_{3} \times \mathfrak{H}_{3} \times \mathfrak{H}_{2}$ gives further relations. Together these suffice to determine all the coefficients $b\left(I_{8}(\Delta), T\right)$ for $T$ a positive definite half-integral symmetric matrix with diagonal equal to $(1, \ldots, 1)$. At the same time it gives us a number of Fourier coefficients of the generator $G \in S_{10}\left(\Gamma_{6}\right)$. This shows that we get a non-zero

\begin{tabular}{|c|c|c|c|c|c|c|c|c|c|}
\hline \multicolumn{2}{|r|}{$2 T_{i}$} & $b\left(I_{8}(\Delta), T_{i}\right)$ & \multicolumn{2}{|r|}{$2 T_{i}$} & $b\left(I_{8}(\Delta), T_{i}\right)$ & \multicolumn{2}{|r|}{$2 T_{i}$} & \multicolumn{2}{|c|}{$b\left(I_{8}(\Delta), T_{i}\right)$} \\
\hline \multirow{2}{*}{\multicolumn{2}{|c|}{$\begin{array}{r}8 A_{1} \\
5 A_{1} \oplus A_{3}\end{array}$}} & 146657280 & & $6 A_{1} \oplus A_{2}$ & 9676800 & \multicolumn{2}{|c|}{$4 A_{1} \oplus 2 A_{2}$} & & 3456000 \\
\hline & & -1612800 & & $A_{1} \oplus 3 A_{2}$ & 362880 & \multicolumn{2}{|c|}{$3 A_{1} \oplus A_{2} \oplus A_{3}$} & & 311040 \\
\hline \multicolumn{2}{|c|}{$4 A_{2}$} & 1970568 & & $4 A_{1} \oplus A_{4}$ & -760320 & \multicolumn{2}{|c|}{$A_{1} \oplus 2 A_{2} \oplus A_{3}$} & & -293760 \\
\hline \multicolumn{2}{|c|}{$2 A_{1} \oplus 2 A_{3}$} & 393728 & & $4 A_{1} \oplus D_{4}$ & -523776 & \multicolumn{2}{|c|}{$2 A_{1} \oplus A_{2} \oplus A_{4}$} & & -51840 \\
\hline \multicolumn{2}{|c|}{$A_{2} \oplus 2 A_{3}$} & -126720 & $2 A_{1} \in$ & $A_{2} \oplus A_{4}$ & -34560 & \multicolumn{2}{|c|}{$3 A_{1} \oplus A_{5}$} & & -34560 \\
\hline \multicolumn{2}{|c|}{$2 A_{2} \oplus A_{4}$} & 146880 & $A_{1} €$ & $A_{3} \oplus A_{4}$ & 23520 & \multicolumn{2}{|c|}{$2 A_{2} \oplus D_{4}$} & & 86400 \\
\hline \multicolumn{2}{|c|}{$A_{1} \oplus A_{2} \oplus A_{5}$} & -41328 & $A_{1} \ominus$ & $A_{3} \oplus D_{4}$ & 5760 & \multicolumn{2}{|c|}{$3 A_{1} \oplus D_{5}$} & & 5760 \\
\hline \multicolumn{2}{|c|}{$2 A_{2} \oplus A_{6}$} & 13440 & & $2 A_{4}$ & 17330 & \multicolumn{2}{|c|}{$A_{3} \oplus D_{5}$} & & -12960 \\
\hline \multicolumn{2}{|c|}{$A_{1} \oplus A_{2} \oplus D_{5}$} & -12960 & & $A_{2} \oplus A_{6}$ & 5040 & \multicolumn{2}{|c|}{$A_{4} \oplus D_{4}$} & & 8640 \\
\hline & $2 D_{4}$ & 4416 & & $A_{3} \oplus D_{5}$ & -4288 & \multicolumn{2}{|c|}{$A_{1} \oplus A_{7}$} & & -704 \\
\hline $2 A_{1}$ & $D_{6}$ & 3392 & & $A_{2} \oplus D_{6}$ & 1440 & \multicolumn{2}{|c|}{$2 A_{1} \oplus E_{6}$} & & 1440 \\
\hline$A_{2}$ & $E_{6}$ & 1440 & & $A_{8}$ & 9 & \multicolumn{2}{|c|}{$A_{1} \oplus D_{7}$} & & -240 \\
\hline & $D_{8}$ & 8 & & $A_{1} \oplus E_{7}$ & -56 & \multicolumn{2}{|c|}{$E_{8}$} & & 1 \\
\hline $2 N$ & $a(G$, & & $2 N$ & $a(G, N)$ & $2 N$ & $a(G, N)$ & & $N$ & $a(G, N)$ \\
\hline $6 A_{1}$ & 280 & $4 A_{1}$ & $\oplus A_{2}$ & -15744 & $2 A_{1} \oplus 2 A_{2}$ & 8496 & $3 A_{1} \oplus$ & & 10176 \\
\hline $3 A_{2}$ & & $A_{1} \oplus A_{2}$ & $\oplus A_{3}$ & 2472 & $2 A_{1} \oplus A_{4}$ & 1000 & $2 A_{1} \oplus$ & & 384 \\
\hline $2 A_{3}$ & -1 & $A_{2}$ & $\oplus A_{4}$ & 750 & $A_{1} \oplus A_{5}$ & -164 & $A_{2} \oplus$ & & 384 \\
\hline$A_{1} \oplus D_{5}$ & & 52 & $A_{6}$ & 7 & $D_{6}$ & 2 & & $E_{6}$ & 1 \\
\hline
\end{tabular}
cusp form of weight 10 on $\Gamma_{6}$. The results are given in two tables. 
Proposition 7.1. The restriction of second order of $I_{8}(\Delta)$ along $\mathfrak{H}_{7} \times \mathfrak{H}_{1}$ is a non-zero form in $S_{2,0,0,0,0,0,10}\left(\Gamma_{7}\right) \otimes S_{12}\left(\Gamma_{1}\right)$. The restriction of the generator $G \in S_{10}\left(\Gamma_{6}\right)$ along $\mathfrak{H}_{5} \times \tilde{H}_{1}$ is a non-zero form in $S_{2,0,0,0,10}\left(\Gamma_{5}\right) \otimes$ $S_{12}\left(\Gamma_{1}\right)$.

Proof. We write the term $t_{2}\left(I_{8}(\Delta)\right)$ as $F \otimes \Delta$ with $F \in S_{2,0,0,0,0,0,10}\left(\Gamma_{7}\right)$. It is given as the transpose of

$$
\left.\left(\frac{\partial^{2} I_{8}(\Delta)}{\partial \tau_{18}^{2}}, 2 \frac{\partial^{2} I_{8}(\Delta)}{\partial \tau_{18} \tau_{28}}, \ldots, \frac{\partial^{2} I_{8}(\Delta)}{\partial \tau_{78}^{2}}\right)\right|_{\mathfrak{H}_{7} \times \mathfrak{H}_{1}}
$$

Then the Fourier expansion of $F$ starts as follows:

$$
F(\tau)=\sum_{N>0} a(N) e^{2 \pi i \operatorname{Tr}(N \tau)}=\sum_{N \in \mathcal{I}_{7}} P_{N}\left(q_{a b}, q_{a b}^{-1}\right) \cdot q_{1} \cdots q_{7}+\ldots
$$

where

$\tau=\left(\tau_{i j}\right) \in \mathfrak{H}_{7}, \quad q_{a}=e^{2 \pi i \tau_{a a}}, \quad q_{a b}=e^{2 \pi i \tau_{a b}}, \quad P_{N}\left(X, X^{-1}\right) \in \mathbb{C}\left[X, X^{-1}\right]^{28}$

and $\mathcal{I}_{7}$ is the set of symmetric positive definite half-integral $7 \times 7$ matrices with $(1, \ldots, 1)$ on the diagonal. The 'constant' term in the Fourier expansion of $F$ is $a\left(1_{7}\right)$ and we get it by substituting $\tau_{18}=\ldots=\tau_{78}=0$ in the transpose of

$$
\left(\frac{\partial^{2} P}{\partial \tau_{18}^{2}}, 2 \frac{\partial^{2} P}{\partial \tau_{18} \tau_{28}}, \ldots, \frac{\partial^{2} P}{\partial \tau_{78}^{2}}\right)
$$

where

$$
P=\sum_{N \in \mathcal{I}_{8}} b\left(I_{8}(\Delta), N\right) q_{18}^{n_{18}} \cdots q_{78}^{n_{78}}
$$

and $\mathcal{I}_{8}$ is the set of positive definite half-integral matrices of the form $\left(\begin{array}{ll}1_{7} & n \\ n^{t} & 1_{1}\end{array}\right)$. This set of matrices contains 379 elements and we can classify them modulo $\mathrm{GL}(7, \mathbb{Z})$-equivalence. As it turns out the lattices $8 A_{1}(1 / 2)$, $6 A_{1}(1 / 2) \oplus A_{2}(1 / 2), \quad 5 A_{1}(1 / 2) \oplus A_{3}(1 / 2)$ and $4 A_{1}(1 / 2) \oplus D_{4}(1 / 2)$ occur 
with multiplicities 1, 14, 84 and 280. We thus find

$$
\begin{aligned}
P= & b\left(I_{8}(\Delta), 8 A_{1}(1 / 2)\right) \\
& +b\left(I_{8}(\Delta), 6 A_{1}(1 / 2) \oplus A_{2}(1 / 2)\right)\left(q_{18}+q_{18}^{-1}+\ldots+q_{78}+q_{78}^{-1}\right) \\
& +b\left(I_{8}(\Delta), 5 A_{1}(1 / 2) \oplus A_{3}(1 / 2)\right)\left(q_{18} q_{28}+\ldots+q_{18} q_{78}+\ldots\right) \\
& +b\left(I_{8}(\Delta), 4 A_{1}(1 / 2) \oplus D_{4}(1 / 2)\right)\left(q_{18} q_{28} q_{38}+\ldots+q_{18} q_{68} q_{78}+\ldots\right) \\
= & 146657280+9676800\left(q_{18}+q_{18}^{-1}+\ldots+q_{78}+q_{78}^{-1}\right) \\
& -1612800\left(q_{18} q_{28}+\ldots+q_{18} q_{78}+\ldots\right) \\
& -523776\left(q_{18} q_{28} q_{38}+\ldots+q_{18} q_{68} q_{78}+\ldots\right) .
\end{aligned}
$$

Thus we get that the vector ${ }^{t} a\left(1_{7}\right)$ equals $\pi^{2} \cdot 82206720$ times

$$
[1,0,0,0,0,0,0,1,0,0,0,0,0,1,0,0,0,0,1,0,0,0,1,0,0,1,0,1]
$$

which shows our result for $I_{8}(\Delta)$. For the restriction of $G$ the argument is similar. Instead of $\mathcal{I}_{8}$ we have a set $\mathcal{I}_{6}$ of 131 elements representing the lattices $6 A_{1}(1 / 2), 4 A_{1}(1 / 2) \oplus A_{2}(1 / 2), 3 A_{1}(1 / 2) \oplus A_{3}(1 / 2)$ and $2 A_{1}(1 / 2) \oplus$ $D_{4}(1 / 2)$ with multiplicities $1,10,40$ and 80 from which we find for the constant term $a\left(1_{5}\right)$ the transpose of

$$
-4 \pi^{2} 149760[1,0,0,0,0,1,0,0,0,1,0,0,1,0,1]
$$

Along similar lines one finds the following result.

Proposition 7.2. The second order restriction $t_{2}(G)$ of the generator $G \in S_{10}\left(\Gamma_{6}\right)$ along $\mathfrak{H}_{5} \times \mathfrak{H}_{1}$ is a non-zero form in $S_{2,0,0,0,10}\left(\Gamma_{5}\right) \otimes S_{12}\left(\Gamma_{1}\right)$. Furthermore, the second order restriction of $G$ along $\mathfrak{H}_{3} \times \mathfrak{H}_{3}$ yields a form $f \otimes f$ with $f$ a non-zero form in $S_{2,0,10}\left(\Gamma_{3}\right)$.

Note that the spaces $S_{2,0,0,0,10}\left(\Gamma_{5}\right)$ and $S_{2,0,10}\left(\Gamma_{3}\right)$ are both 1-dimensional according to Taïbi [20].

\section{The Sibling $\chi_{6,8}$ of degree 2}

As we have seen the form $\chi_{6,8}$ in $S_{6,8}\left(\Gamma_{2}\right)$ appears ubiquitously. Its presence was first seen in the cohomology of local systems on the moduli spaces $\mathcal{A}_{2}$ and $\mathcal{M}_{2}$ in [8]. One of us asked Ibukiyama whether he could construct a form in $S_{6,8}\left(\Gamma_{2}\right)$. Ibukiyama answered in 2001 with a construction 
of this form using theta functions with pluriharmonic polynomial coefficients. Now we have easier ways to construct it. One way is as follows. Let $G_{i}^{t}=\left(\partial \vartheta_{i} / \partial z_{1}, \partial \vartheta_{i} / \partial z_{2}\right)$ be the (transposed) gradient of the $i$ th odd theta function for $i=1, \ldots, 6$, see [7]. It defines a section of $\mathbb{E}_{2} \otimes \operatorname{det}\left(\mathbb{E}_{2}\right)^{1 / 2}$ for the congruence subgroup $\Gamma_{2}[4,8]$. We let $\operatorname{Sym}^{j}\left(\mathbb{E}_{2}\right)$ be the $\mathfrak{S}_{j}$-invariant subbundle of $\mathbb{E}_{2}^{\otimes j}$. Then the expression

$$
\operatorname{Sym}^{j}\left(G_{1}, \ldots, G_{6}\right)
$$

defines a cusp form $f_{6,3}$ of weight $(6,3)$ on the principal congruence subgroup $\Gamma_{2}[2]$. The product $\chi_{5} f_{6,3}$ with $\chi_{5}$, the product of the ten even theta characteristics, is a form of level 1 and is up to a normalization equal to $\chi_{6,8}$ :

$$
\chi_{6,8}:=-\left(\chi_{5} \operatorname{Sym}^{6}\left(G_{1}, \ldots, G_{6}\right)\right) / 4096 \pi^{6}
$$

We write its Fourier expansion as

$$
\chi_{6,8}(\tau)=\sum_{N>0} a(N) e^{2 \pi i \operatorname{Tr}(N \tau)}=\sum_{N>0}{ }^{t}\left(a(N)_{0}, \ldots, a(N)_{6}\right) e^{2 \pi i \operatorname{Tr}(N \tau)} .
$$

It starts as follows (with $q_{1}=e^{2 \pi i \tau_{1}}, q_{2}=e^{2 \pi i \tau_{2}}$ and $r=e^{2 \pi i \tau_{12}}$ )

$$
\begin{aligned}
& \left(\begin{array}{c}
0 \\
0 \\
r^{-1}-2+r \\
2\left(r-r^{-1}\right) \\
r^{-1}-2+r \\
0 \\
0
\end{array}\right) q_{1} q_{2}+\left(\begin{array}{c}
0 \\
0 \\
-2\left(r^{-2}+8 r^{-1}-18+8 r+r^{2}\right) \\
8\left(r^{-2}+4 r^{-1}-4 r-r^{2}\right) \\
-2\left(7 r^{-2}-4 r^{-1}-6-4 r+7 r^{2}\right) \\
12\left(r^{-2}-2 r^{-1}+2 r-r^{2}\right) \\
-4\left(r^{-2}-4 r^{-1}+6-4 r+r^{2}\right)
\end{array}\right) q_{1} q_{2}^{2} \\
& +\left(\begin{array}{c}
-4\left(r^{-2}-4 r^{-1}+6-4 r+r^{2}\right) \\
12\left(r^{-2}-2 r^{-1}+2 r-r^{2}\right) \\
-2\left(7 r^{-2}-4 r^{-1}-6-4 r+7 r^{2}\right) \\
8\left(r^{-2}+4 r^{-1}-4 r-r^{2}\right) \\
-2\left(r^{-2}+8 r^{-1}-18+8 r+r^{2}\right) \\
0 \\
0
\end{array}\right) q_{1}^{2} q_{2}+\left(\begin{array}{c}
16\left(r^{-3}-9 r^{-1}+16-9 r+r^{3}\right) \\
-72\left(r^{-3}-3 r^{-1}+3 r-r^{3}\right) \\
+128\left(r^{-3}-2+r^{3}\right) \\
-144\left(r^{-3}+5 r^{-1}-5 r-r^{3}\right) \\
+128\left(r^{-3}-2+r^{3}\right) \\
-72\left(r^{-3}-3 r^{-1}+3 r-r^{3}\right) \\
16\left(r^{-3}-9 r^{-1}+16-9 r+r^{3}\right)
\end{array}\right) q_{1}^{2} q_{2}^{2}+\ldots
\end{aligned}
$$

Using standard involutions one sees that interchanging $q_{1}$ and $q_{2}$ inverts the order of the coordinates of $a(N)$, while interchanging $r$ and $1 / r$ makes the $i$ th coordinate change sign by $(-1)^{i}$ for $i=0, \ldots, 6$. One can read off the first non-zero Fourier coefficients:

\begin{tabular}{|r|r|r|}
\hline${ }^{t} a([1,0,1])$ & ${ }^{t} a([1,1,1])$ & ${ }^{t} a([1,0,2])$ \\
\hline$(0,0,-2,0,-2,0,0)$ & $(0,0,1,2,1,0,0)$ & $(0,0,36,0,12,0,-24)$ \\
\hline
\end{tabular}


Remark 8.1. We can play the same game of restriction also with $\chi_{6,8}$. It restriction to $\mathfrak{H}_{1} \times \mathfrak{H}_{1}$ vanishes. Its first non-vanishing term is $t_{1}\left(\chi_{6,8}\right)$ and it can be viewed as a section of

$$
\oplus_{i=0}^{6} L_{1}^{15-i} \otimes L_{2}^{9+i}
$$

with $L_{1}$ and $L_{2}$ the Hodge bundle $\mathbb{E}_{1}$ on the first and second component. It turns out to be $(0,0,0, \Delta \otimes \Delta, 0,0,0)$.

The question arises what the zero locus of $\chi_{6,8}$ is. It contains $\mathcal{A}_{1,1}$. Probably this is all. We prove that additionally there are only finitely many points where $\chi_{6,8}$ vanishes.

Proposition 8.2. The zero locus of $\chi_{6,8}$ in $\mathcal{A}_{2}$ consists of $\mathcal{A}_{1,1}$ and possibly finitely many isolated points.

Proof. If $V$ is the zero locus of $\chi_{6,8}$ in $\mathcal{A}_{2}$ then $V$ contains $\mathcal{A}_{1,1}$. Let $V_{1}$ be the 1-dimensional part of $V$. Then the closure $\bar{V}_{1}$ of $V_{1}$ in the Satake compactification $\mathcal{A}_{2}^{*}$ intersects the closure $\overline{\mathcal{A}}_{1,1}$ of $\mathcal{A}_{1,1}$ since it is an ample divisor. But in the neighborhood of $\overline{\mathcal{A}}_{1,1}$ our form is given by $(0,0,0, \Delta \otimes \Delta, 0,0,0)$ and this does not vanish in $U \backslash \overline{\mathcal{A}}_{1,1}$ with $U$ a suitable open neighborhood of $\overline{\mathcal{A}}_{1,1}$ in $\mathcal{A}_{2}^{*}$. Hence every irreducible component of the curve $V_{1}$ has to intersect $\overline{\mathcal{A}}_{1,1}$ at the point $(\infty, \infty)$, the 0 -dimensional cusp of $\mathcal{A}_{2}^{*}$. But there the Fourier series shows that $\chi_{6,8}$ does not vanish in $U^{\prime} \backslash\left(U^{\prime} \cap \overline{\mathcal{A}}_{1,1}\right)$ for $U^{\prime}$ a suitable neighborhood of the 0 -dimensional cusp of $\mathcal{A}_{2}^{*}$.

We end this section by giving a list of eigenvalues $\lambda_{p}$ for the Hecke operator $T_{p}$ for $p$ prime $\leq 47$. These eigenvalues were obtained by using the Fourier expansion. In an appendix we give a short summary how to calculate such eigenvalues. In [8] the eigenvalues $\lambda_{p}$ of $T(p)$ and $\lambda_{p^{2}}$ of $T\left(p^{2}\right)$ were calculated for $p=2,3,5$ and 7 using a completely different method, namely using counting points on genus 2 curves over finite fields. Also the eigenvalues of all $T(q)$ for $q \leq 37$ and $q \neq 27$ are available by [8], see also [3]. These values agree. Yet another way to obtain these eigenvalues is given in [5, Section 2, Table 3] where Chenevier and Lannes use the Kneser neighborhoods of lattices to calculate these. 


\begin{tabular}{|r|r|r|}
\hline$p$ & $\lambda_{p}$ & $\lambda_{p^{2}}$ \\
\hline 2 & 0 & -57344 \\
3 & -27000 & 143765361 \\
5 & 2843100 & -7734928874375 \\
7 & -107822000 & 4057621173384801 \\
11 & 3760397784 & \\
13 & 9952079500 & \\
17 & 243132070500 & \\
19 & 595569231400 & \\
23 & -6848349930000 & \\
29 & 53451678149100 & \\
31 & 234734887975744 & \\
37 & 448712646713500 & \\
43 & -1828093644641000 & \\
47 & -6797312934516000 & \\
\hline
\end{tabular}

\section{The Sibling in $S_{4,0,8}\left(\Gamma_{3}\right)$}

Let $B$ (beta) denote a generator of the 1-dimensional space $S_{4,0,8}\left(\Gamma_{3}\right)$. We can find $B$ in the restriction $B \otimes \Delta$ of the Schottky form $J_{8}$ along $\mathfrak{H}_{3} \times \tilde{H}_{1}$. This enables us to write the beginning of the Fourier expansion. With the variables $q_{m}=e^{2 \pi i \tau_{m}}$ and $u=e^{2 \pi i \tau_{12}}, v=e^{2 \pi i \tau_{13}}, w=e^{2 \pi i \tau_{23}}$, we find the expansion

$$
B(\tau)=\left(\begin{array}{c}
0 \\
0 \\
0 \\
(v-1)^{2}(w-1)^{2} / v w \\
(u-1)(v-1)(w-1)(-1+1 / v w+1 / u w-1 / u v) \\
(u-1)^{2}(w-1)^{2} / u w \\
0 \\
(u-1)(v-1)(w-1)(-1+1 / v w-1 / u w+1 / u v) \\
(u-1)(v-1)(w-1)(-1-1 / v w+1 / u w+1 / u v) \\
0 \\
0 \\
0 \\
(u-1)^{2}(v-1)^{2} / u v \\
0 \\
0
\end{array}\right) q_{1} q_{2} q_{3}+\cdots
$$

Using the action of $\gamma \in \Gamma_{3}$ that sends $\tau_{i j}$ to $\tau^{\prime}=\tau_{\sigma(i), \sigma(j)}$ with $\sigma=(13) \in \mathfrak{S}_{3}$ we see that the coordinates $v_{i}(i=1, \ldots, 15)$ of the Fourier coefficients satisfy the identities $v_{1}(\tau)=v_{15}\left(\tau^{\prime}\right), v_{2}(\tau)=v_{14}\left(\tau^{\prime}\right), v_{3}(\tau)=v_{10}\left(\tau^{\prime}\right), v_{4}(\tau)=$ $v_{13}\left(\tau^{\prime}\right), v_{5}(\tau)=v_{9}\left(\tau^{\prime}\right), v_{6}(\tau)=v_{6}\left(\tau^{\prime}\right), v_{7}(\tau)=v_{12}\left(\tau^{\prime}\right), v_{8}(\tau)=v_{8}\left(\tau^{\prime}\right)$ One 
can use the Fourier coefficients

$$
\begin{aligned}
& a([111 ; 000])={ }^{t}[0,0,0,4,0,4,0,0,0,0,0,0,4,0,0] \\
& a([222 ; 000])={ }^{t}[512,0,0,2816,0,2816,0,0,0,0,512,0,2816,0,512] \\
& a([112 ; 122])={ }^{t}[0,0,0,0,1,1,0,1,3,2,0,0,1,2,1] \\
& a([1222 ; 020])={ }^{t}[0,0,0,-24,0,-48,0,-48,0,-96,48,0,-48,0,-48] \\
& a([122 ; 200])={ }^{t}[0,0,0,-48,0,-24,-96,0,-48,0,-48,0,-48,0,48] \text {; }
\end{aligned}
$$

to calculate the Hecke eigenvalue at 2 , see the appendix. We have $\lambda_{2}=$ -1728 , and this fits because $B$ is a lift of $\Delta$ from $\Gamma_{1}$ (see [3]) and its Hecke eigenvalue $\lambda_{p}$ is given by the formula

$$
\lambda_{p}=\tau(p)\left(p^{5}+\tau(p)+p^{6}\right),
$$

where $\tau(p)$ is the Hecke eigenvalue of $\Delta$ at $p$.

\section{Appendix: Hecke Operators for Vector valued Modular forms of Degree Two}

Here we give a short treatment of Hecke operators on vector-valued Siegel modular forms of degree 2 . The basic reference is [2].

\subsection{The Hecke operator $T_{p}$}

For a prime $p$ we consider the double coset $T_{p}=\Gamma_{2} \operatorname{diag}(1,1, p, p) \Gamma_{2}$. Following Andrianov, we have the following left coset decomposition for $T_{p}$.

Proposition 10.1. The double coset $T_{p}$ admits the following left coset decomposition:

$$
\begin{aligned}
& \Gamma_{2}\left(\begin{array}{llll}
p & 0 & 0 & 0 \\
0 & p & 0 & 0 \\
0 & 0 & 1 & 0 \\
0 & 0 & 0 & 1
\end{array}\right)+\sum_{0 \leq a, b, c \leq p-1} \Gamma_{2}\left(\begin{array}{cccc}
1 & 0 & a & b \\
0 & 1 & b & c \\
0 & 0 & p & 0 \\
0 & 0 & 0 & p
\end{array}\right) \\
& +\sum_{0 \leq a \leq p-1} \Gamma_{2}\left(\begin{array}{cccc}
0 & -p & 0 & 0 \\
1 & 0 & a & 0 \\
0 & 0 & 0 & -1 \\
0 & 0 & p & 0
\end{array}\right)+\sum_{0 \leq a, m \leq p-1} \Gamma_{2}\left(\begin{array}{cccc}
p & 0 & 0 & 0 \\
-m & 1 & 0 & a \\
0 & 0 & 1 & m \\
0 & 0 & 0 & p
\end{array}\right)
\end{aligned}
$$

and we have $\operatorname{deg}\left(T_{p}\right)=p^{3}+p^{2}+p+1$. 
Then we define an action of $T_{p}$ on $M_{j, k}\left(\Gamma_{2}\right)$ via

$$
\left.F\right|_{j, k} T_{p}=\left.p^{2 k+j-3} \sum_{i} F\right|_{j, k} \sigma_{i}
$$

where for $\sigma_{i}=\left(\begin{array}{ll}a_{i} & b_{i} \\ c_{i} & d_{i}\end{array}\right)$, the slash operator is given by:

$$
\left(\left.F\right|_{j, k} \sigma_{i}\right)(\tau)=\operatorname{det}\left(c_{i} \tau+d_{i}\right)^{-k} \operatorname{Sym}^{j}\left(\left(c_{i} \tau+d_{i}\right)^{-1}\right) F\left(\left(a_{i} \tau+b_{i}\right)\left(c_{i} \tau+d_{i}\right)^{-1}\right)
$$

for every $\tau \in \mathfrak{H}_{2}$.

The action on the Fourier expansion of $F(\tau)=\sum_{N \geq 0} a(N) e^{2 \pi i \operatorname{Tr}(N \tau)}$ is given by the following proposition. If

$$
\left(\left.F\right|_{j, k} T_{p}\right)(\tau)=\sum_{N \geqslant 0} b(N) e^{2 \pi i \operatorname{Tr}(N \tau)}
$$

and if we write $\left[n_{1}, n_{12}, n_{2}\right]$ for $N=\left(\begin{array}{cc}n_{1} & n_{12} / 2 \\ n_{12} / 2 & n_{2}\end{array}\right)$ with $n_{i} \in \mathbb{N}$ and $n_{12} \in \mathbb{Z}$, we have

Proposition 10.2. The coefficient $b\left(\left[n_{1}, n_{12}, n_{2}\right]\right)$ is given by

$$
\begin{aligned}
& p^{2 k+j-3} a\left(\left[\frac{n_{1}}{p}, \frac{n_{12}}{p}, \frac{n_{2}}{p}\right]\right)+a\left(\left[p n_{1}, p n_{12}, p n_{2}\right]\right) \\
& +p^{k+j-2} \operatorname{Sym}^{j}\left(\begin{array}{cc}
0 & 1 / p \\
-1 & 0
\end{array}\right) a\left(\left[\frac{n_{2}}{p},-n_{12}, p n_{1}\right]\right) \\
& +p^{k+j-2} \sum_{0 \leq m \leq p-1} \operatorname{Sym}^{j}\left(\begin{array}{cc}
1 & -m / p \\
0 & 1 / p
\end{array}\right) a\left(\left[\frac{n_{1}+n_{12} m+n_{2} m^{2}}{p}, n_{12}+2 n_{2} m, p n_{2}\right]\right)
\end{aligned}
$$

where $a\left(\left[n_{1}, n_{12}, n_{2}\right]\right)=0$ if $n_{1}, n_{2}$ and $n_{12}$ are not all integral.

Corollary 10.3. For $N=[1,1,1]$ the Fourier coefficient $b([1,1,1])$ is given by $a([p, p, p])+$

$$
\begin{cases}3^{k-2} \operatorname{Sym}^{j}\left(\begin{array}{cc}
3 & -1 \\
0 & 1
\end{array}\right) a([1,3,3]) & \text { if } p=3 \\
0 & \text { if } p \not \equiv 1 \bmod 3 \\
p^{k-2} \sum_{i=1}^{2} \operatorname{Sym}^{j}\left(\begin{array}{cc}
p-m_{i} \\
0 & 1
\end{array}\right) a\left(\left[\frac{1+m_{i}+m_{i}^{2}}{p}, 1+2 m_{i}, p\right]\right) & \text { if } p \equiv 1 \bmod 3\end{cases}
$$

where in the latter case $m_{1}$ and $m_{2}$ are the two integers $0 \leq m_{i} \leq p-1$ that reduce to the roots of the polynomial $1+X+X^{2}$ over $\mathbb{F}_{p}$. 
Corollary 10.4. For $N=[1,0,1]$, the coefficient $b([1,0,1])$ is given by

$$
\begin{cases}a([2,0,2])+2^{k-2} \operatorname{Sym}^{j}\left(\begin{array}{cc}
2 & -1 \\
0 & 1
\end{array}\right) a([1,2,2]) & \text { if } p=2 \\
a([p, 0, p]) & \text { if } p \neq \equiv 1 \bmod 4 \\
a([p, 0, p])+p^{k-2} \operatorname{Sym}^{j}\left(\begin{array}{cc}
p \pm m_{0} \\
0 & 1
\end{array}\right) a\left(\left[\frac{1+m_{0}^{2}}{p}, \mp 2 m_{0}, p\right]\right) & \text { if } p \equiv 1 \bmod 4\end{cases}
$$

where in the latter case $\pm m_{0}$ are the two roots of the polynomial $1+X^{2}$ over $\mathbb{F}_{p}$.

With these corollaries we can calculate eigenvalues $\lambda_{p}$ of an eigenform $F \in M_{j, k}\left(\Gamma_{2}\right)$ as follows. If $p \neq 3$ and $p \not \equiv 1(\bmod 3)$ and the $i$ th coordinate of the vector $a([1,1,1])$ does not vanish then we can take $\lambda_{p}=$ $a([p, p, p])_{i} / a([1,1,1])_{i}$, and similarly, for $p \neq 2$ and $p \not \equiv 1(\bmod 4)$ we can take $\lambda_{p}=a([p, p, p])_{i} / a([1,0,1])_{i}$ provided that the $i$ th coordinate of $a([1,0,1])$ does not vanish. Moreover, if the last component of $a([1,1,1])$ is not zero and $p=3$ or $p \equiv 1(\bmod 3)$ we can use only this component to get $\lambda_{p}$ since $\operatorname{Sym}^{j}\left(\begin{array}{ll}p & * \\ 0 & 1\end{array}\right)$ is upper triangular. Similarly, if the last component of $a([1,0,1])$ is not zero and $p=2$ or $p \equiv 1 \bmod 4$, we can use only this component in order to compute $\lambda_{p}$ for the same reason as before.

\subsection{The Hecke operator $T_{p^{2}}$}

The Hecke operator $T_{p^{2}}$ is defined via the double coset

$$
T_{p^{2}}=\Gamma_{2}\left(\begin{array}{cccc}
p & 0 & 0 & 0 \\
0 & p & 0 & 0 \\
0 & 0 & p & 0 \\
0 & 0 & 0 & p
\end{array}\right) \Gamma_{2}+\Gamma_{2}\left(\begin{array}{cccc}
1 & 0 & 0 & 0 \\
0 & p & 0 & 0 \\
0 & 0 & p^{2} & 0 \\
0 & 0 & 0 & p
\end{array}\right) \Gamma_{2}+\Gamma_{2}\left(\begin{array}{cccc}
1 & 0 & 0 & 0 \\
0 & 1 & 0 & 0 \\
0 & 0 & p^{2} & 0 \\
0 & 0 & 0 & p^{2}
\end{array}\right) \Gamma_{2}
$$

Proposition 10.5. The Hecke operator $T_{p^{2}}$ has degree $p^{6}+p^{5}+2 p^{4}+$ $2 p^{3}+p^{2}+p+1$ and admits the following left coset decomposition

$$
\begin{aligned}
& \Gamma_{2}\left(\begin{array}{cccc}
p^{2} & 0 & 0 & 0 \\
0 & p^{2} & 0 & 0 \\
0 & 0 & 1 & 0 \\
0 & 0 & 0 & 1
\end{array}\right)+\sum_{0 \leq a, b, c \leq p-1} \Gamma_{2}\left(\begin{array}{cccc}
p & 0 & a & b \\
0 & p & b & b \\
0 & 0 & p & 0 \\
0 & 0 & 0 & 0
\end{array}\right)+\sum_{0 \leq a, b, c \leq p^{2}-1} \Gamma_{2}\left(\begin{array}{cccc}
1 & 0 & a & b \\
0 & 1 & b & c \\
0 & 0 & p^{2} & 0 \\
0 & 0 & 0 & p^{2}
\end{array}\right) \\
& +\sum_{0 \leq a \leq p-1} \Gamma_{2}\left(\begin{array}{cccc}
p & 0 & a & 0 \\
0 & p^{2} & 0 & 0 \\
0 & 0 & p & 0 \\
0 & 0 & 0 & 1
\end{array}\right)+\sum_{0 \leq a, m \leq p-1} \Gamma_{2}\left(\begin{array}{ccccc}
p^{2} & 0 & 0 & 0 \\
-m & p & p & 0 & a \\
0 & 0 & 0 & 0 \\
0 & 0 & 0 & p
\end{array}\right)+\sum_{0 \leq a, b \leq p-1} \Gamma_{2}\left(\begin{array}{cccc}
1 & 0 & c & -b \\
0 & p & -p & a \\
0 & 0 & 0 & 0 \\
0 & 0 & 0 & 0 \\
0 & 0 & p
\end{array}\right)
\end{aligned}
$$

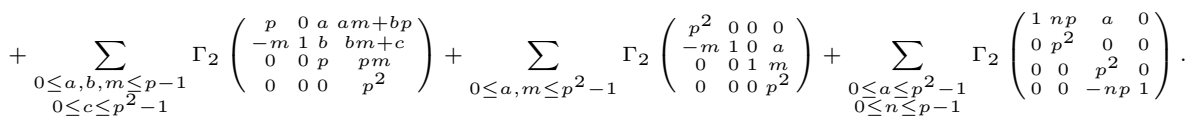


We now consider the action on a modular form $F \in M_{j, k}\left(\Gamma_{2}\right)$ defined by

$$
\left.F\right|_{j, k} T_{p^{2}}=\left.p^{4 k+2 j-6} \sum_{i} F\right|_{j, k} \sigma_{i}
$$

where the sum is over the left coset representatives. If $F=$ $\sum_{N \geq 0} a(N) e^{2 \pi i \operatorname{Tr}(N \tau)}$ the result is a modular form with Fourier expansion $\sum_{N \geq 0} c(N) e^{2 \pi i \operatorname{Tr}(N \tau)}$ with the $c(N)=a_{p^{2}}(N)$ expressed in the $a(N)$ as follows.

Proposition 10.6. Let $F \in M_{j, k}\left(\Gamma_{2}\right)$ with Fourier expansion

$F(\tau)=\sum_{N \geqslant 0} a(N) e^{2 \pi i \operatorname{Tr}(N \tau)} \quad$ and write $\left.\quad F\right|_{j, k} T_{p^{2}}(\tau)=\sum_{N \geqslant 0} a_{p^{2}}(N) e^{2 \pi i \operatorname{Tr}(N \tau)}$.

Then we have with $N=\left[n_{1}, n_{12}, n_{2}\right]$ that $a_{p^{2}}(N)$ is equal to

$a\left(p^{2} N\right)+p^{4 k+2 j-6} a\left(\frac{N}{p^{2}}\right)+p^{2 k+j-3} a(N) \delta_{p}\left(n_{1}, n_{12}, n_{2}\right)$

$+p^{3 k+j-5} \operatorname{Sym}^{j}\left(\begin{array}{ll}1 & 0 \\ 0 & p\end{array}\right) a\left(\left[n_{1}, n_{12} / p, n_{2} / p^{2}\right]\right) \delta_{p}\left(n_{1}\right)$

$+p^{3 k+j-5} \sum_{0 \leq m \leq p-1} \operatorname{Sym}^{j}\left(\begin{array}{cc}p & -m \\ 0 & 1\end{array}\right) a\left(\left[\frac{n_{1}+n_{12} m+n_{2} m^{2}}{p^{2}}, \frac{n_{12}+2 n_{2} m}{p}, n_{2}\right]\right) \delta_{p}\left(n_{2}\right)$

$+p^{k-2} \operatorname{Sym}^{j}\left(\begin{array}{ll}1 & 0 \\ 0 & p\end{array}\right) a\left(\left[p^{2} n_{1}, p n_{12}, n_{2}\right]\right) \delta_{p}\left(n_{2}\right)$ $+p^{k-2} \sum_{\substack{0 \leq m \leq p-1 \\ p \mid\left(n_{1}+n_{12} m+n_{2} m^{2}\right)}} \operatorname{Sym}^{j}\left(\begin{array}{cc}p-m \\ 0 & 1\end{array}\right) a\left(\left[n_{1}+n_{12} m+n_{2} m^{2}, p\left(n_{12}+2 n_{2} m\right), p^{2} n_{2}\right]\right)$

$+p^{2 k-4} \sum_{0 \leq m \leq p^{2}-1} \operatorname{Sym}^{j}\left(\begin{array}{c}p^{2}-m \\ 0 \\ 1\end{array}\right) a\left(\left[\frac{n_{1}+n_{12} m+n_{2} m^{2}}{p^{2}}, n_{12}+2 n_{2} m, p^{2} n_{2}\right]\right)$

$+p^{2 k-4} \sum_{0 \leq n \leq p-1} \operatorname{Sym}^{j}\left(\begin{array}{cc}1 & 0 \\ n p & p^{2}\end{array}\right) a\left(\left[p^{2} n_{1}, n_{12}-2 p n_{1} n, \frac{n_{1} p^{2} n^{2}-n_{12} n p+n_{2}}{p^{2}}\right]\right)$

where $a\left(\left[n_{1}, n_{12}, n_{2}\right]\right)=0$ if $n_{1}, n_{12}$ and $n_{2}$ are not all integral.

From this proposition, we deduce the following corollary: 
Corollary 10.7. Let $F \in M_{j, k}\left(\Gamma_{2}\right)$ be a Hecke eigenform with eigenvalue $\lambda_{p^{2}}$ at $p^{2}$. Assume that $a([1,1,1]) \neq 0$. Then $\lambda_{p^{2}}(F) a([1,1,1])$ equals

$$
\begin{aligned}
& a\left(p^{2} N\right)+p^{k-2} \sum_{\substack{0 \leq m \leq p-1 \\
1+m+m^{2} \equiv 0 \bmod p}} \operatorname{Sym}^{j}\left(\begin{array}{cc}
p & -m \\
0 & 1
\end{array}\right) a\left(\left[1+m+m^{2}, p(1+2 m), p^{2}\right]\right) \\
&+p^{2 k-4} \sum_{0 \leq m \leq p^{2}-1} \operatorname{Sym}^{j}\left(\begin{array}{cc}
p^{2} & -m \\
0 & 1
\end{array}\right) a\left(\left[\frac{1+m+m^{2}}{p^{2}}, 1+2 m, p^{2}\right]\right) .
\end{aligned}
$$

\section{Appendix: Hecke Operators for Vector valued Modular forms of Degree Three}

Here we treat only the case of the Hecke operator $T_{p}$ for $p$ a prime. Here $T_{p}$ is the double coset $\Gamma_{3} \operatorname{diag}(1,1,1, p, p, p) \Gamma_{3}$.

Proposition 11.1. We have $\operatorname{deg}\left(T_{p}\right)=p^{6}+p^{5}+p^{4}+2 p^{3}+p^{2}+p+1$ and there is the following left coset decomposition.

$$
\begin{aligned}
& \Gamma_{3}\left(\begin{array}{llllll}
p & 0 & 0 & 0 & 0 & 0 \\
0 & p & 0 & 0 & 0 & 0 \\
0 & 0 & p & 0 & 0 & 0 \\
0 & 0 & 0 & 1 & 0 & 0 \\
0 & 0 & 0 & 0 & 1 & 0 \\
0 & 0 & 0 & 0 & 0 & 1
\end{array}\right)+\sum_{0 \leq a, \ldots, f \leq p-1} \Gamma_{3}\left(\begin{array}{llllll}
1 & 0 & 0 & a & b & c \\
0 & 1 & 0 & b & d & e \\
0 & 0 & 0 & c & c & e \\
0 & 0 & 0 & p & 0 & 0 \\
0 & 0 & 0 & 0 & 0 & 0 \\
0 & 0 & 0 & 0 & 0 & p
\end{array}\right)+ \\
& \sum_{0 \leq a, u, v \leq p-1} \Gamma_{3}\left(\begin{array}{cccccc}
p & 0 & 0 & 0 & 0 & 0 \\
0 & p & 0 & 0 & 0 & 0 \\
-u & -v & 1 & 0 & 0 & a \\
0 & 0 & 0 & 1 & 0 & 0 \\
0 & 0 & 0 & 0 & 1 & 0 \\
0 & 0 & 0 & 0 & 0 & p
\end{array}\right)+\sum_{0 \leq a, u \leq p-1} \Gamma_{3}\left(\begin{array}{cccccc}
p & 0 & 0 & 0 & 0 & 0 \\
0 & 0 & p & 0 & 0 & 0 \\
-u & 1 & 0 & 0 & a & 0 \\
0 & 0 & 0 & 1 & u & 0 \\
0 & 0 & 0 & 0 & 0 & 1 \\
0 & 0 & 0 & 0 & 0 & 0
\end{array}\right) \\
& +\sum_{0 \leq a \leq p-1} \Gamma_{3}\left(\begin{array}{cccccc}
0 & 0 & p & 0 & 0 & 0 \\
0 & p & 0 & 0 & 0 & 0 \\
1 & 0 & 0 & a & 0 & 0 \\
0 & 0 & 0 & 0 & 0 \\
0 & 0 & 0 & 0 & 0 & 0 \\
0 & 0 & 0 & 0 & 0 & 0
\end{array}\right)+\sum_{0 \leq a, b, c, u, v \leq p-1} \Gamma_{3}\left(\begin{array}{cccccc}
p & 0 & 0 & 0 & 0 & 0 \\
- & 1 & 0 & 0 & 0 & b \\
-v & 0 & 1 & 0 & 0 & 0 \\
0 & 0 & 0 & 1 & c \\
0 & 0 & 0 & 0 & 0 \\
0 & 0 & 0 & 0 & 0 \\
0 & 0 & 0 & 0 & p
\end{array}\right)
\end{aligned}
$$

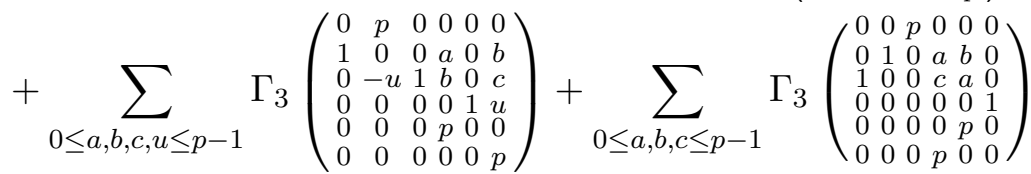

We are now able to describe the action of the operator $T_{p}$ on the Fourier expansion of a modular form on $\Gamma_{3}$. Let $F \in M_{i, j, k}\left(\Gamma_{3}\right)$ with Fourier expansion $F(\tau)=\sum_{N \geq 0} a(N) e^{2 \pi i \operatorname{Tr}(N \tau)}$. The action of the operator $T_{p}$ on $F$ reads as follows:

$$
\left.F\right|_{i, j, k} T_{p}=\left.p^{i+2 j+3 k-6} \sum_{i} F\right|_{i, j, k} \sigma_{i}
$$


where for $\sigma_{i}=\left(\begin{array}{ll}a_{i} & b_{i} \\ c_{i} & d_{i}\end{array}\right)$, the slash operator is given by:

$$
\begin{array}{r}
\left.\left(\left.F\right|_{i, j, k} \sigma_{i}\right)(\tau)=\operatorname{det}\left(c_{i} \tau+d_{i}\right)^{-k} \operatorname{Sym}^{i}\left(\left(c_{i} \tau+d_{i}\right)^{-1}\right) \operatorname{Sym}^{j}\left(\wedge^{2}\left(\left(c_{i} \tau+d_{i}\right)^{-1}\right)\right)\right) \\
\cdot F\left(\left(a_{i} \tau+b_{i}\right)\left(c_{i} \tau+d_{i}\right)^{-1}\right)
\end{array}
$$

for every $\tau \in \mathfrak{H}_{3}$. Let us write

$$
\left(\left.F\right|_{i, j, k} T_{p}\right)(\tau)=\sum_{N \geqslant 0} a_{p}(N) e^{2 \pi i \operatorname{Tr}(N \tau)} .
$$

We will use two kinds of notations for $N=\left(\begin{array}{ccc}n_{1} & n_{12} / 2 & n_{13} / 2 \\ n_{12} / 2 & n_{2} & n_{23} / 2 \\ n_{13} / 2 & n_{23} / 2 & n_{3}\end{array}\right)$, namely

$$
N=\left(\begin{array}{ccc}
n_{1} & n_{12} / 2 & n_{13} / 2 \\
n_{12} / 2 & n_{2} & n_{23} / 2 \\
n_{13} / 2 & n_{23} / 2 & n_{3}
\end{array}\right) \leftrightarrow\left[n_{1} n_{2} n_{3} ; n_{12} n_{13} n_{23}\right] \leftrightarrow\left[\begin{array}{c}
n_{1} \\
n_{2} \\
n_{3} \\
n_{13} \\
n_{23} \\
n_{23}
\end{array}\right] .
$$

We also use the notations

$$
\operatorname{Sym}^{i}\left(a^{-1}\right)=\operatorname{Sym}^{-i}(a) \text { and } \operatorname{Sym}^{j}\left(\wedge^{2}\left(a^{-1}\right)\right)=\operatorname{Sym}^{-j}\left(\wedge^{2}(a)\right) .
$$

Proposition 11.2. We have that $a_{p}(N)$ is given by

$$
\begin{aligned}
& p^{i+2 j+3 k-6} a(N / p)+a(p N) \\
& +p^{i+2 j+2 k-5} \sum_{0 \leq u, v \leq p-1} \operatorname{Sym}^{-i}\left(\begin{array}{lll}
1 & 0 & u \\
0 & 1 & 0 \\
0 & 0 & p
\end{array}\right) \operatorname{Sym}^{-j}\left(\wedge^{2}\left(\left(\begin{array}{lll}
1 & 0 & u \\
0 & 1 & 0 \\
0 & 0 & p
\end{array}\right)\right) a\left(\left[\begin{array}{c}
\left(n_{1}+n_{13} u+n_{3} u^{2}\right) / p \\
\left(n_{2}+n_{23} 3_{3} v+n_{3} v^{2}\right) / p \\
\left(n_{12}+n_{23} u+n_{1} n_{13} v+2 n_{3} u v\right) / p \\
n_{13}+2 n_{3} u \\
n_{23}+2 n_{3} v
\end{array}\right]\right)\right. \\
& +p^{i+2 j+2 k-5} \sum_{0 \leq u \leq p-1} \operatorname{Sym}^{-i}\left(\begin{array}{lll}
1 & u & 0 \\
0 & 0 & 1 \\
0 & p & 0
\end{array}\right) \operatorname{Sym}^{-j}\left(\wedge^{2}\left(\left(\begin{array}{lll}
1 & u & 0 \\
0 & 0 & 1 \\
0 & p & 0
\end{array}\right)\right) a\left(\left[\begin{array}{c}
\left(n_{1}+n_{12} u+n_{2} u^{2}\right) / p \\
n_{3} / p \\
p n_{2} \\
\left(n_{13}+2 n_{23} u\right) / p \\
n_{12}+2 n_{2} u \\
n_{23} u
\end{array}\right]\right)\right. \\
& +p^{i+2 j+2 k-5} \operatorname{Sym}^{-i}\left(\begin{array}{ccc}
0 & 0 & 1 \\
0 & 1 & 0 \\
p & 0 & 0
\end{array}\right) \operatorname{Sym}^{-j}\left(\wedge^{2}\left(\left(\begin{array}{ccc}
0 & 0 & 1 \\
0 & 1 & 0 \\
p & 0 & 0
\end{array}\right)\right) a\left(\left[n_{3} / p n_{2} / p p n_{1} ; n_{23} / p n_{13} / p n_{23}\right]\right)\right. \\
& +p^{i+2 j+k-3} \sum_{0 \leq u, v \leq p-1} \operatorname{Sym}^{-i}\left(\begin{array}{ccc}
1 & u & v \\
0 & 0 & 0 \\
0 & 0 & p
\end{array}\right) \operatorname{Sym}^{-j}\left(\wedge^{2}\left(\left(\begin{array}{ccc}
1 & u & v \\
0 & p & 0 \\
0 & 0 & p
\end{array}\right)\right) a\left(\left[\begin{array}{c}
\left(n_{1}+n_{12} u+n_{13} v+n_{2} u^{2}+n_{23} u v+n_{3} v^{2}\right) / p \\
p p n_{2} \\
p n_{3} \\
n_{12}+2 n_{2} u+n_{23} v \\
n_{13}+n_{23} u+2 n_{3} v \\
p n_{23}
\end{array}\right]\right)\right. \\
& +p^{i+2 j+k-3} \sum_{0 \leq u \leq p-1} \operatorname{Sym}^{-i}\left(\begin{array}{ccc}
0 & 1 & u \\
p & 0 & 0 \\
0 & 0 & p
\end{array}\right) \operatorname{Sym}^{-j}\left(\wedge^{2}\left(\left(\begin{array}{ccc}
0 & 1 & u \\
0 & 0 & 0 \\
0 & 0 & p
\end{array}\right)\right) a\left(\left[\begin{array}{c}
\left(n_{2}+n_{23} u+n_{3} u^{2}\right) / p \\
p \\
p n_{1} \\
n_{3} \\
n_{12}+n_{13} \\
n_{23} n_{2}+2 n_{3} u \\
p \\
n_{13}
\end{array}\right]\right)\right. \\
& +p^{i+2 j+k-3} \operatorname{Sym}^{-i}\left(\begin{array}{ccc}
0 & 0 & 1 \\
0 & 0 & 0 \\
p & 0 & 0
\end{array}\right) \operatorname{Sym}^{-j}\left(\wedge^{2}\left(\left(\begin{array}{ccc}
0 & 0 & 1 \\
0 & p & 0 \\
p & 0 & 0
\end{array}\right)\right) a\left(\left[n_{3} / p p n_{2} p n_{1} ; n_{23} n_{13} p n_{12}\right]\right)\right.
\end{aligned}
$$

where $a\left(\left[n_{1} n_{2} n_{3} ; n_{12} n_{13} n_{23}\right]\right)=0$ if $n_{1}, \ldots, n_{23}$ are not all integral. 


\section{References}

[1] A. Andrianov: Hecke operators and quadratic forms. Grundlehren der Mathematischen Wissenschaften 286. Springer-Verlag, Berlin, 1987.

[2] A. Andrianov: Euler products corresponding to Siegel modular forms of genus 2. Russian Math. Surveys 29, (1974), 45-116.

[3] J. Bergström, C. Faber, G. van der Geer: Siegel modular forms of degree three and the cohomology of local systems. Selecta Math. 20 (2014), $83-124$.

[4] R. Borcherds, E. Freitag, R. Weissauer: A Siegel cusp form of degree 12 and weight 12. J. Reine Angew. Math. 494 (1998), 141-153.

[5] G. Chenevier: Représentations galoisiennes automorphes et conséquences arithmétiques des conjectures de Langlands et Arthur. arXiv: $1307.5170 \mathrm{v} 1$.

[6] G. Chenevier, D. Renard: Level one algebraic cusp forms of classical groups of small ranks. arXiv:1207.0724

[7] F. Cléry, G. van der Geer, S. Grushevsky: Siegel modular forms of genus 2 and level 2. arXiv:1306.6018. Int. Journal of Math. 26, (2015).

[8] C. Faber, G. van der Geer: Sur la cohomologie des systèmes locaux sur les espaces des modules des courbes de genre 2 et des surfaces abéliennes, I, II. C.R. Acad. Sci. Paris, Sér. I 338 (2004), 381-384, 467-470.

[9] G. van der Geer: Siegel modular forms and their applications. In: J. Bruinier, G. van der Geer, G. Harder, D. Zagier: The 1-2-3 of modular forms. Springer Verlag, 2008.

[10] T. Ibukiyama: On differential operators on automorphic forms and invariant pluri-harmonic polynomials. Comm. Math. Univ. Sancti Pauli 48 (1999), 103-117.

[11] J.I. Igusa: Schottky's invariant and quadratic forms. In: Christoffel Symposium, Birkhauser Verlag, 1981, 352-362.

[12] J.I. Igusa: Problems on abelian functions at the time of Poincaré and some at present. Bull. Amer. Math. Soc. (N.S.) 6 (1982), 161-174.

[13] T. Ikeda: On the lifting of elliptic cusp forms to Siegel cusp forms of degree 2n. Ann. of Math. 154, (2001), 641-681. 
[14] R. Keaton: Eigenvalues of Ikeda lifts. Ramanujan J. 37, (2015), 299-307.

[15] I. Miyawaki: Numerical examples of Siegel cusp forms of degree 3 and their zeta-function, Memoirs of the Faculty of Science, Kyushu University, Ser. A 46, 307-339 (1992)

[16] G. Nebe, B. Venkov: On Siegel modular forms of weight 12. J. Reine Angew. Math. 531 (2001), 49-60.

[17] E. Witt: Eine Identität zwischen Modulformen zweiten Grades. Math. Sem. Hamburg 14 1941, 323-337.

[18] C. Poor, D. Yuen: Computations of spaces of Siegel modular cusp forms. J. Math. Soc. Japan 59, No. 1 (2007), 185-222.

[19] R. Sasaki: Modular forms vanishing at the reducible points of the Siegel upper-half space. J. Reine Angew. Math. 345 (1983), 111-121.

[20] O. Taïbi: Dimensions of spaces of level one automorphic forms for split classical groups using the trace formula. arXiv:1406.4247

[21] S. Tsuyumine: On Siegel modular forms of degree three. Amer. J. Math. 108 (1986), no. 4, 755-862. Addendum to: "On Siegel modular forms of degree three". Amer. J. Math. 108 (1986), no. 4, 1001-1003.

Fabien Cléry

Department Mathematik,

Universität Siegen,

Emmy-Noether-Campus,

Walter-Flex-Strasse 3,

57068 Siegen, Germany.

E-mail: cleryfabien@gmail.com

Gerard van der Geer

Korteweg-de Vries Instituut,

Universiteit van Amsterdam,

Postbus 94248,

1090 GE Amsterdam, The Netherlands.

E-mail: geer@science.uva.nl 
